\title{
Grand symmetry, spectral action and the Higgs mass
}

\author{
Agostino Devastato, ${ }^{a, b}$ Fedele Lizzi $^{a, b, c}$ and Pierre Martinetti ${ }^{a, b}$ \\ ${ }^{a}$ Dipartimento di Fisica, Università di Napoli Federico II, \\ Monte S. Angelo, Via Cintia, 80126 Napoli, Italy \\ ${ }^{b}$ INFN, Sezione di Napoli, \\ Monte S. Angelo, Via Cintia, 80126 Napoli, Italy \\ ${ }^{c}$ Departament de Estructura $i$ Constituents de la Matèria, Institut de Ciéncies del Cosmos, \\ Universitat de Barcelona, Barcelona, Catalonia, Spain \\ E-mail: agostino.devastato@na.infn.it, fedele.lizzi@na.infn.it, \\ martinetti.pierre@gmail.com
}

ABSTRACT: In the context of the spectral action and the noncommutative geometry approach to the standard model, we build a model based on a larger symmetry. With this grand symmetry it is natural to have the scalar field necessary to obtain the Higgs mass in the vicinity of $126 \mathrm{GeV}$. This larger symmetry mixes gauge and spin degrees of freedom without introducing extra fermions. Requiring the noncommutative space to be an almost commutative geometry (i.e. the product of manifold by a finite dimensional internal space) gives conditions for the breaking of this grand symmetry to the standard model.

KeYwords: Non-Commutative Geometry, Higgs Physics, Beyond Standard Model, GUT

ARXIV EPRINT: 1304.0415 


\section{Contents}

1 Introduction 1

2 The spectral triple of the standard model 3

2.1 Spectral triples 3

2.2 Hilbert space of the standard model 4

2.3 Dirac operator 5

2.4 Spectral action, Higgs mass and the $\sigma$ field 5

3 Algebras and representations $\quad 7$

$\begin{array}{ll}3.1 & \text { The algebra of the standard model }\end{array}$

$\begin{array}{lr}3.2 & \text { The grand algebra }\end{array}$

$\begin{array}{lll}3.3 & \text { Reduction due to grading } & 11\end{array}$

4 The Majorana coupling and the $\sigma$ field $\quad 12$

$\begin{array}{lll}4.1 & \text { Dirac operator with Majorana mass term } & 12\end{array}$

$\begin{array}{lll}4.2 & \text { First order condition for Majorana Dirac operator } & 14\end{array}$

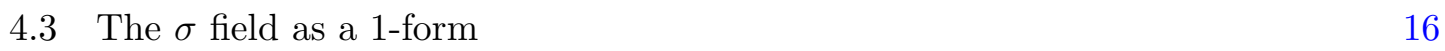

5 Reduction to the standard model $\quad \mathbf{1 7}$

5.1 First order condition for the free Dirac operator 18

$\begin{array}{ll}5.2 \text { Emergence of spin } & 19\end{array}$

$\begin{array}{lll}5.3 & \text { Fiat neutrino } & 20\end{array}$

6 Conclusions and outlook $\quad 20$

$\begin{array}{ll}\text { A Reduction of the } \mathcal{A}_{\mathcal{F}} \text { algebra } & 22\end{array}$

B Fluctuation of $D_{R}$ by the standard model algebra 24

\section{Introduction}

Noncommutative geometry [1-4] generalizes the concepts of ordinary geometry in an algebraic setting and enables powerful generalizations beyond the Riemannian paradigm. Its application to the standard model of fundamental interactions is a fascinating one [5-10]: the geometrical setting is that of an usual manifold (spacetime) described by the algebra of complex valued functions defined on it, tensor multiplied by a finite dimensional matrix algebra. This is usually called an "almost commutative geometry". The standard model is described as a particular almost commutative geometry, and the corresponding Lagrangian is built from the spectrum of a generalized Dirac operator. This approach to the standard 
model has a phenomenological predictive power and is approaching the level of maturity which enables it to confront with experiments.

Schematically the application of noncommutative geometry to the standard model has two sides. One is the mathematical request that a topological space is a manifold. This yields a set of algebraic requirements [11] involving the algebra of functions defined on the space, represented as bounded operators on a spinorial Hilbert space, and a (generalized) Dirac operator, plus two more operators representing charge conjugation and chirality. These requirements, being algebraic, can easily be generalized to noncommutative algebra [12]. In the almost commutative case some extra assumptions on the representation [13] single out the algebra corresponding to the standard model among a restricted number of cases $[9,14]$ as the smallest algebra which satisfies the requirements.

The other side has to do with the spectral nature of the action. The spectral action principle [15] allows to derive from a unique noncommutative spacetime a Lagrangian for both general relativity (in Euclidean signature) and the standard model. The principle is purely spectral, based on the regularization of the eigenvalues of the Dirac operator. ${ }^{1}$ In [9] (see also $[14,21]$ ) this noncommutative model was enhanced to include massive neutrinos and the seesaw mechanism. The most remarkable result is the possibility to predict the mass of the Higgs particle from the mass of the other fermions and the value of the unification scale. Earlier version of the model had a prediction around $170 \mathrm{GeV}$, a value ruled out by Tevatron in 2008. Recently Connes and Chamseddine showed in [22] that the experimental value of the mass of Higgs at $126 \mathrm{GeV}$ can be obtained introducing a new scalar field $\sigma$ suitably coupled to the Higgs field. Such a field had previously been proposed from a completely different perspective by particle physicists (see for example [23]), to avoid an high energy instability [24-26] in the Higgs potential.

The idea of a new scalar field to lower the mass of the Higgs in the Connes approach is not new, and was already proposed by Stephan in [27]. However, he obtained it adding new fermions [28, 29], whereas in [22] (as well as in the present paper) the fermionic contents of the standard model is not touched. In [22] the field $\sigma$ is obtained by simply turning one of the elements of the internal Dirac operator into a field. As explained in section 2.4, this is somehow artificial because the usual NCG procedure to obtain scalar fields (the so called fluctuations of the metric) does not work for the field $\sigma$, because of one of the conditions on spectral triples (the first order condition). In section 4.3 we show how to overcome this difficulty by considering a larger algebra. ${ }^{2}$ This is the main result of the paper.

More precisely, in [13] it is shown that under minimal condition on the representation of the algebra, the smallest nontrivial almost commutative manifold corresponds to the standard model. Here we consider a larger algebra, that we term grand algebra. We show how to obtain the field $\sigma$ by fluctuating the Majorana mass term of the Dirac operator, in a way compatible with the first order condition induced by this Majorana mass term. Then

\footnotetext{
${ }^{1}$ The spectral action principle, as well as any finite mode regularization [16-18], requires a Euclidean compact spacetime, but the cutoff on the momentum eigenvalues is even more general and can be used also for continuum spectrum, see for example [19, 20].

${ }^{2}$ While we were preparing the second version of this paper, a proposal came out to obtain the $\sigma$ field from a fluctuation of the metric, by relaxing the first order condition [30, 31].
} 
we show how the first order condition imposed by the free Dirac operator reduces the grand algebra to the one of the standard model. The field $\sigma$ then appears as the Higgs-like field corresponding to this reduction. All this is possible because we intertwine in a non trivial way the Riemann-spin degrees of freedom (the components of spinors) with the internal degrees of freedom (the particles of the standard model). This puts in a new light also the phenomenon of fermion doubling [9, 32-34] present in the theory.

The paper is organized as follows. In section 2 we briefly recall the spectral triple construction (section 2.1) and introduce the Hilbert space (section 2.2) and the Dirac operator (section 2.3) of the standard model. We recall in section 2.4 how the Higgs mass is obtained from the spectral action, and point out the difficulty in generating the field $\sigma$ by fluctuation of the metric. Section 3 deals with the choice of the algebras and their representation. We first discuss the algebra of the standard model in section 3.1, then introduce the grand algebra in section 3.2. The reduction imposed by the grading condition are worked out in section 3.3. In section 4 we explain how the grand algebra allows to obtain the field $\sigma$ : first we work out the most general Dirac operator $D_{\nu}$ compatible with the grand algebra and containing a Majorana mass term for the neutrino (section 4.1), then we calculate the reduction imposed by the first order condition induced by $D_{\nu}$ (section 4.2), finally we show that $\sigma$ can be obtained by a fluctuation of $D_{\nu}$ respecting this first order condition (section 4.3). Section 5 deals with the reduction of the grand algebra to the algebra of the standard model (section 5.1) and the issue of Lorentz invariance and the emergence of the spin structure (section 5.2). Possible physical interpretations are discussed in section 5.3. A final section contains conclusions and some speculative comments.

\section{The spectral triple of the standard model}

\section{$2.1 \quad$ Spectral triples}

The basic device in the construction is a spectral triple $(\mathcal{A}, \mathcal{H}, D)$ consisting of a $*$-algebra $\mathcal{A}$ of bounded operators in a Hilbert space $\mathcal{H}$ - containing the identity operator - and a nonnecessarily bounded self-adjoint operator $D$. These three elements satisfy a set of properties allowing to prove Connes reconstruction theorem: given any spectral triple $(\mathcal{A}, \mathcal{H}, D)$ with commutative $\mathcal{A}$ satisfying the required conditions, then $\mathcal{A} \simeq C^{\infty}(\mathcal{M})$ for some Riemannian spin manifold $\mathcal{M}$. The required conditions can be found in [11], and their noncommutative generalization in [12]. In this work we will be interested only in

- the grading condition: there is an operator $\Gamma$ (called chirality) such that $\Gamma^{2}=\mathbb{I}$, $\Gamma D=-D \Gamma$ and

$$
[\Gamma, a]=0 \quad \forall a \in \mathcal{A} .
$$

- the order zero condition: there is an antilinear isometry $J$ (called real structure) which implements an action of the opposite algebra ${ }^{3} \mathcal{A}^{\circ}$ obtained by identifying $b^{\circ}=J b^{*} J^{-1}$, and which commutes with the action of $\mathcal{A}$ :

$$
\left[a, J b J^{-1}\right]=0 \quad \forall a, b \in \mathcal{A} .
$$

\footnotetext{
${ }^{3}$ Identical to $\mathcal{A}$ as a vector space, but with reversed product: $a^{\circ} b^{\circ}=(b a)^{\circ}$.
} 
The operator $J$ must obey 1) $J^{2}= \pm I$; 2) $J D= \pm D J$; 3) $J \Gamma= \pm \Gamma J$, with choice of signs dictated by the $K O$-dimension of the spectral triple.

- the first order condition

$$
\left[[D, a], J b J^{-1}\right]=0 \quad \forall a, b \in \mathcal{A} .
$$

\subsection{Hilbert space of the standard model}

A particular form of noncommutative manifolds, suitable to describe the standard model of elementary particles [12], are the almost commutative geometries, given by the product of an ordinary manifold $\mathcal{M}$ (that from now on is assumed to have dimension 4 ) by a finite dimensional spectral triple. The algebra is

$$
\mathcal{A}=C^{\infty}(\mathcal{M}) \otimes \mathcal{A}_{F}
$$

where $\mathcal{A}_{F}$ is a finite dimensional algebra, whose choice is dictated by the gauge group of the standard model and is discussed in section 3. For the Hilbert space a suitable choice is

$$
\mathcal{H}=\operatorname{sp}\left(L^{2}(\mathcal{M})\right) \otimes \mathcal{H}_{F}
$$

where $s p\left(L^{2}(\mathcal{M})\right)$ is the Hilbert space of square summable spinors on $\mathcal{M}$ and

$$
\mathcal{H}_{F}=\mathcal{H}_{R} \oplus \mathcal{H}_{L} \oplus \mathcal{H}_{R}^{c} \oplus \mathcal{H}_{L}^{c}=C^{96}
$$

contains all the 96 particle-degrees of freedom of the standard model: 8 fermions (electron, neutrino, up and down quarks with three colours each) for $\mathrm{N}=3$ families and 2 chiralities $\left(\mathcal{H}_{R} \simeq \mathcal{H}_{L} \simeq C^{24}\right)$ plus antiparticles $\left(\mathcal{H}_{R}^{c} \simeq \mathcal{H}_{L}^{c} \simeq C^{24}\right)$. The chiral and real structure are

$$
\Gamma=\gamma^{5} \otimes \gamma_{F}, \quad J=\mathcal{J} \otimes J_{F}
$$

where $\mathcal{J}$ is the charge conjugation operator, $\gamma^{5}$ the product of the four $\gamma$ matrices, and

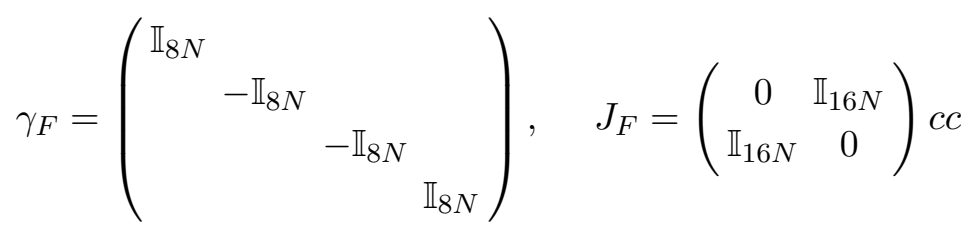

with $c c$ the complex conjugation. Notice that right particles and left antiparticles have chirality +1 , whereas left particles and right antiparticles have chirality -1 .

The Hilbert space $\mathcal{H}$ defined in (2.5) is the tensor product of four dimensional spinors by the 96 -dimensional elements of $\mathcal{H}_{F}$, thus (as a vector bundle over $\mathcal{M}$ ) it has dimension 384 , or 128 for a single generation. This redundancy of states is known as fermion doubling [3234]. The problem is not only the over-counting, but the presence of states which do not have a definite parity, being left chiral in the inner indices and right chiral in the outer ones, or viceversa. Since the total chirality $\Gamma$ is the product of $\gamma_{F}$ (which acts on the inner indices of $\mathcal{H}_{F}$ ) by $\gamma$ (which acts on the spin indices), the spurious states are the ones for 
which $\Gamma \Psi=-\Psi$. Taking the functional integral of the fermionic action to be a Pfaffian [9] allows to project out these extra degrees of freedom. However, one cannot simply project out the extra states and work with a representation on a smaller Hilbert space, because in the bosonic spectral action all degrees of freedom are necessary [32] in order to obtain the proper action of the standard model coupled with gravity. We will see in the following that the fermion doubling may be in fact an essential feature of the model, by allowing to represent an algebra bigger than the one of the standard model.

\subsection{Dirac operator}

The operator $D$ (still called Dirac operator) for the spectral triple of the standard model is

$$
D=\not \partial \otimes \mathbb{I}_{96}+\gamma^{5} \otimes D_{F}
$$

with $^{4}$

$$
D_{F}=\left(\begin{array}{cccc}
0_{8 N} & \mathcal{M} & \mathcal{M}_{R} & 0_{8 N} \\
\mathcal{M}^{\dagger} & 0_{8 N} & 0_{8 N} & 0_{8 N} \\
\mathcal{M}_{R}^{\dagger} & 0_{8 N} & 0_{8 N} & \overline{\mathcal{M}} \\
0_{8 N} & 0_{8 N} & \mathcal{M}^{T} & 0_{8 N}
\end{array}\right) .
$$

The matrix $\mathcal{M}$ contains the Yukawa couplings of the fermions and the mixing matrices (CKM for quarks and NPMS for neutrinos). It couples left with right particles. The matrix $\mathcal{M}_{R}=\mathcal{M}_{R}^{T}$ contains Majorana masses and couples right particles with right antiparticles.

The operators $\gamma_{F}, J_{F}$ and $D_{F}$ are such that

$$
J_{F}^{2}=I, \quad J_{F} D_{F}=D_{F} J_{F}, \quad J \gamma_{F}=-\gamma_{F} J_{F},
$$

meaning that the finite part of the spectral triple has $K O$-dimension $6[9,21]$. The manifold part has $K O$-dimension 4 , and the full spectral triple has $K O$-dimension $6+4=10 \bmod 8=2$.

\subsection{Spectral action, Higgs mass and the $\sigma$ field}

Given an almost commutative geometry $(\mathcal{A}, \mathcal{H}, \mathcal{D})$, a fluctuation of the metric $[12]^{5}$ means the substitution of $D$ by the gauge Dirac operator [36]

$$
D_{A} \equiv D+A+J A J^{-1}
$$

where $A=\sum_{i} a_{i}\left[D, b_{i}\right]$, with $a_{i}, b_{i} \in \mathcal{A}$, is a generalized gauge potential. It is made of two parts: a scalar field on $\mathcal{M}$ with value in $\mathcal{A}_{F}$, and 1-form field on $\mathcal{M}$ with value in the group of unitaries of $\mathcal{A}_{F}$. In case $\mathcal{A}_{F}=\mathcal{A}_{s m}$ is the algebra of the standard model (discussed in section 3), the 1-form fields yield the vector bosons mediating the three fundamental interactions, and the scalar field is the Higgs field $H$.

The spectral action [15] is based on a regularization of the spectrum of $D_{A}$. It reads:

$$
S_{B}=\operatorname{Tr} \chi\left(\frac{D_{A}^{2}}{\Lambda^{2}}\right)
$$

\footnotetext{
${ }^{4}$ Here $^{-}$denotes the complex conjugation, $\dagger$ the adjoint, ${ }^{T}$ the transpose.

${ }^{5}$ The name comes from the fact that the substitution $D \rightarrow D_{A}$ modifies the metric associated to the spectral triple. See [35] for a detailed account on this point.
} 
where $\chi$ is a cutoff function, usually the (smoothened) characteristic function on the interval $[0,1]$, and $\Lambda$ is a renormalization scale. It has an expansion in power series of $\Lambda^{-1}$,

$$
\lim _{\Lambda \rightarrow \infty} S_{B}=\sum_{n} f_{n} a_{n}\left(D_{A}^{2} / \Lambda^{2}\right)
$$

where the $f_{n}$ are the momenta of $\chi$ and the $a_{n}$ are the Seeley-de Witt coefficients [37, 38].

Applied to the operator (2.9) fluctuated as in (2.12) with $a_{i}, b_{i} \in C^{\infty}(\mathcal{M}) \otimes \mathcal{A}_{s m}$, the expansion (2.14) yields the bosonic part of the Lagrangian of the standard model coupled with gravity [9, section 4.1]. ${ }^{6}$ Furthermore the parameters related to the Higgs come out to be function of the parameters in $D_{F}$, i.e. the Yukawa couplings, which are in turn dominated by the top quark coupling. In this sense the model predicts the Higgs mass as a function of the other gauge couplings, the Yukawa top mass and the scale $\Lambda$ which appears also as the scale in which the three gauge couplings coincide. This last point is known to be true only in an approximate sense. If one takes the unification scale to be $\Lambda=10^{17} \mathrm{GeV}$ then one finds - assuming the big desert hypothesis - a Higgs mass of the order of $170 \mathrm{GeV}$. This value is not in agreement with the recent LHC experiments [46, 47].

One can think of extending the model to solve this. There have been several proposals in this sense, and some of them are reviewed in [8]. In particular C. Stephan has proposed in [27] that the presence of an extra scalar field, corresponding to the breaking of a extra $\mathrm{U}(1)$ symmetry, can bring down the mass of the Higgs to $126 \mathrm{GeV}$. This model however contains extra fermions. Earlier examples of extensions are in [28, 29, 48-52].

Recently, in [22] the noncommutative geometry model was enhanced to also overcome the high energies instability of a Higgs boson with mass around $126 \mathrm{GeV}$, in addition to predicting the correct mass. This is done ruling out the hypothesis of the "big desert" and considering an additional scalar field $\sigma$ that lives at high energies and gives mass to the Majorana neutrinos. Explicitly $\sigma$ is obtained in [22] by turning (inside the finite dimensional part $D_{F}$ of the Dirac operator) the constant-entry $k_{R}$ of the Majorana matrix $\mathcal{M}_{R}$ into a field:

$$
k_{R} \rightarrow k_{R} \sigma
$$

However, the origin of the field $\sigma$ is quite different from the Higgs. The latter, like the other bosons, are components of the gauge potential $A$. They are obtained from the commutator of $D_{F}$ with the algebra: $D_{F}$ has constant components, that is without space dependence, but when these numbers are commuted with elements of the algebra they give rise to the desired bosonic fields. One could hope to obtain $\sigma$ in a similar way, by considering $k_{R}$ as a Yukawa coupling. As explained in appendix $\mathrm{B}$, the problem is that in taking the commutator with elements of the algebra $\mathcal{A}_{s m}$, the coefficient $k_{R}$ does not contribute to the potential because of the first order condition. This forced the authors of [22] to "promote to a field" only the entry $k_{R}$, in a somewhat arbitrary way. Indeed the components of $D_{F}$ cannot all be fields to start with, otherwise the model would loose

\footnotetext{
${ }^{6}$ The bosonic action can also be obtained via considerations related to spectral regularization and the role of anomalies [39-41]. Supersymmetric extension have been investigated in [42]. For some cosmological predictions based on the spectral action, see e.g. [43-45].
} 
its predictive power, in that all Yukawa couplings would be fields, and the masses of all fermions would run independently, thus making any prediction impossible. In the following (sections 3 and 4) we show that there is a way to obtain the field $\sigma$ from $k_{R}$ by a fluctuation of the metric, provided one starts with an algebra larger than the one of the standard model.

\section{Algebras and representations}

Under assumptions on the representation (irreducibility, existence of a separating vector), the most general finite algebra in (2.4) that satisfies all conditions for the noncommutative space to be a manifold is

$$
\mathcal{A}_{F}=\mathbb{M}_{a}(\mathbb{H}) \oplus \mathbb{M}_{2 a}(\mathbb{C}) \quad a \in \mathbb{N}^{*}
$$

This algebra acts on an Hilbert space of dimension $2(2 a)^{2}[13,53]$.

\subsection{The algebra of the standard model}

To have a non trivial grading on $\mathbb{M}_{a}(\mathbb{H})$ the integer $a$ must be at least 2 , meaning the simplest possibility is

$$
\mathcal{A}_{\mathcal{F}}=\mathbb{M}_{2}(\mathbb{H}) \oplus \mathbb{M}_{4}(\mathbb{C}) .
$$

Hence an Hilbert space of dimension $2(2 \cdot 2)^{2}=32$, that is the dimension of $\mathcal{H}_{F}$ for one generation. The grading condition $[a, \Gamma]=0$ reduces the algebra to the left-right algebra:

$$
\mathcal{A}_{L R}=\mathbb{H}_{L} \oplus \mathbb{H}_{R} \oplus \mathbb{M}_{4}(\mathbb{C})
$$

This is basically a Pati-Salam model [54], one of the not many models allowed by the spectral action [55]. The order one condition reduces further the algebra to [9] (for a review see also [10])

$$
\mathcal{A}_{s m}=\mathbb{C} \oplus \mathbb{H} \oplus \mathbb{M}_{3}(\mathbb{C}),
$$

where $\mathbb{H}$ are the quaternions, which we represent as $2 \times 2$ matrices, and $\mathbb{M}_{3}(\mathbb{C})$ are $3 \times 3$ complex valued matrices. $\mathcal{A}_{s m}$ is the algebra of the standard model, that is the one whose unimodular group is $\mathrm{U}(1) \times \mathrm{SU}(2) \times \mathrm{U}(3)$. The details of these reductions are given in appendix A.

These algebras - tensorized by $C^{\infty}(\mathcal{M})$ - are represented on the Hilbert space (2.5), whose elements are 384 components vectors. The number 384 comes from degrees of freedom which have different physical meaning. Some of them refer to "internal" degrees of freedom, like colour, some refer to the Riemannian-spin structure, and have a spacetime meaning. We denote a generic fermion, i.e. an element of $\mathcal{H}$ by

$$
\Psi_{s \dot{s} \alpha}^{\mathrm{CI} m}(x) \in \mathcal{H}=L^{2}(\mathcal{M}) \otimes \mathrm{H}_{F}=s p\left(L^{2}(\mathcal{M})\right) \otimes \mathcal{H}_{F} .
$$

The position of the indices, whose meaning is described below, is a matter of convention, $\Psi$ is a $C^{384}$-vector valued function on $\mathcal{M}$, we write some of them as upper indices and some as lower to avoid having six indices in a row. Note the difference between $\mathrm{H}_{F}$ and $\mathcal{H}_{F}$ : the latter is a 96 dimensional space and its vectors are to be multiplied by spinors, while the 
former is the larger 384 dimensional space which exhibits explicitly the fermion doubling over-counting. So far in the literature the Hilbert space has been considered always in its factorized form involving $\mathcal{H}_{F}$. One of the novelties of this work is to use the factorized form involving $\mathrm{H}_{F}$. This allows us in section 3.2 to consider algebras which do not act separately on spinors and the internal part. This means that in addition of the internal degrees of freedom used in [14], our tensorial notation also includes spin indices $s, \dot{s}$.

The meaning and range of the various indices of $\Psi_{s \dot{s} \alpha}^{\mathrm{CI} m}(x)$ is the following:

$s=r, l$
$\dot{s}=\dot{0}, \dot{1}$ are the spinor indices. They are not internal indices in the sense that the algebra $\mathcal{A}_{\mathcal{F}}$ acts diagonally on it. They take two values each, and together they make the four indices on an ordinary Dirac spinor. The index $s=r, l$ indicates chirality and runs over the right, left part of the spinor, while $\dot{s}$ differentiates particles from antiparticles. In the chiral basis one thus has ${ }^{7}$

$$
\gamma^{\mu}=\left(\begin{array}{cc}
0_{2} & \sigma_{\dot{s}}^{\mu \dot{t}} \\
\bar{\sigma}_{\dot{s}}^{\mu \dot{t}} & 0_{2}
\end{array}\right)_{s t}, \quad \gamma^{5}=\left(\begin{array}{cc}
I_{2} & 0_{2} \\
0_{2} & -I_{2}
\end{array}\right)_{s t},
$$

where for $\mu=0,1,2,3$ one defines

$$
\sigma^{\mu}=\left\{I_{2},-i \sigma_{i}\right\}, \quad \bar{\sigma}^{\mu}=\left\{I_{2}, i \sigma_{i}\right\}
$$

with $\sigma_{i}, i=1,2,3$ the Pauli matrices, namely $\sigma^{0}=I_{2}$,

$$
\sigma^{1}=-i \sigma_{1}=\left(\begin{array}{cc}
0 & -i \\
-i & 0
\end{array}\right)_{\dot{s} \dot{t}} \sigma^{2}=-i \sigma_{2}=\left(\begin{array}{cc}
0 & -1 \\
1 & 0
\end{array}\right)_{\dot{s} \dot{t}} \sigma^{3}=-i \sigma_{3}=\left(\begin{array}{cc}
-i & 0 \\
0 & i
\end{array}\right)_{\dot{s} \dot{t}} .
$$

$\mathrm{I}=0, \ldots 3$ indicates a "lepto-colour" index. The zeroth "colour" actually identifies leptons while $\mathrm{I}=1,2,3$ are the usual three colours of QCD.

$\alpha=1 \ldots 4$ is the flavour index. It runs over the set $u_{R}, d_{R}, u_{L}, d_{L}$ when $\mathrm{I}=1,2,3$, and $\nu_{R}, e_{R}, \nu_{L}, e_{L}$ when $\mathrm{I}=0$. It repeats in the obvious way for the other generations.

$C=0,1$ indicates whether we are considering "particles" $(C=0)$ or "antiparticles" $(C=1)$.

$m=1,2,3$ is the generation index. The representation of the algebra of the standard model is diagonal in these indices, the Dirac operator is not, due to Cabibbo-KobayashiMaskawa mixing parameters.

For the remainder of this paper the generation index $m$ does not play any role. We will therefore suppress it and work with one generation, thus effectively considering $\mathcal{H}_{F}$ and $\mathrm{H}_{F}$ having dimension 32 and 128 respectively.

\footnotetext{
${ }^{7}$ The multi-index st after the closing parenthesis is to recall that the entries of the $\gamma$ 's matrices are labelled by indices $s, t$ taking values in the set $\{l, r\}$. For instance the $l$-row, $l$-column block of $\gamma^{5}$ is $\mathbb{I}_{2}$. Similarly the entries of the $\sigma$ 's matrices are labelled by $\dot{s}, \dot{t}$ indices taking value in the set $\{\dot{0}, \dot{1}\}$ : for instance $\sigma_{\dot{0}}^{2^{\dot{0}}}=\sigma_{\dot{1}}^{2 \dot{1}}=0$.
} 
A generic element $A=\{Q, M\}$ in $C^{\infty}(\mathcal{M}) \otimes \mathcal{A}_{\mathcal{F}}\left(\right.$ with $Q \in C^{\infty}(\mathcal{M}) \otimes M_{2}(\mathbb{H})$ and $\left.M \in C^{\infty}(\mathcal{M}) \otimes M_{4}(C)\right)$ acts as a matrix on vectors of $\mathrm{H}_{F}$ with index structure (3.5), it is therefore a matrix with twice as many indices: ${ }^{8}$

$$
A_{s \dot{s} \mathrm{DJ} \alpha}^{t \dot{\mathrm{CI}} \beta}=\delta_{s}^{t} \delta_{\dot{s}}^{\dot{t}}\left(\delta_{0}^{\mathrm{C}} \delta_{\mathrm{J}}^{\mathrm{I}} Q_{\alpha}^{\beta}+\delta_{1}^{\mathrm{C}} M_{\mathrm{J}}^{\mathrm{I}} \delta_{\alpha}^{\beta}\right)
$$

Here $Q_{\alpha}^{\beta}$ evaluated at $x \in \mathcal{M}$ denotes the entries $Q_{\alpha}^{\beta}(x) \in C$ of the matrix $Q(x) \in M_{2}(\mathbb{H})$, viewed as a $4 \times 4$ complex matrix with components labelled by the $\alpha, \beta$ flavour indices. Similarily $M_{\mathrm{J}}^{\mathrm{I}}$ evaluated at $x$ stands for the components of the matrix $M(x) \in M_{4}(\mathbb{C})$, whose entries are labelled by the I, J lepto-colour indices.

The two Kronecker $\delta$ at the beginning of the expression for $A$ show that the algebra acts in a trivial way (i.e. as the identity operator) on the spin indices. In other words the finite dimensional algebra $\mathcal{A}_{\mathcal{F}}$ acts only on the internal indices. The two terms in the bracket act only on particles and antiparticles respectively, as signified by $\delta_{0}^{\mathrm{C}}$ and $\delta_{1}^{\mathrm{C}}$. They are such that the order zero condition hold. Note in fact that for particles the action is trivial on the I, J indices, and for antiparticles is trivial on the $\alpha, \beta$ indices. Since the real structure $J$ exchanges particles with antiparticles the two $A$ and $J B J^{-1}$ will commute. There is no room for the representation of a larger algebra satisfying the order 0 condition, unless more fermions are added, or one renounces to the trivial action on the spin indices. The second possibility is the one we will use for the grand algebra in the following sections.

\subsection{The grand algebra}

The case $a=3$ in (3.1) would require a 72-dimensional Hilbert space, and there is no obvious way to build it from the particle content of the standard model. The next case, $a=4$, requires the Hilbert space to have dimension 128, which is the dimension of $\mathrm{H}_{F}$. Said in an other way, considering together the spin and internal degrees of freedom as part of the "grand Hilbert space" $\mathrm{H}_{F}$ gives precisely the number of dimension to represent the grand algebra

$$
\mathcal{A}_{G}=\mathbb{M}_{4}(\mathbb{H}) \oplus \mathbb{M}_{8}(\mathbb{C}) .
$$

This means that $C^{\infty}(\mathcal{M}) \otimes \mathcal{A}_{G}$ can be represented on the same Hilbert space $\mathcal{H}$ as $C^{\infty}(\mathcal{M}) \otimes$ $\mathcal{A}_{\mathcal{F}}$. The only difference is that one needs to factorize $\mathcal{H}$ in $(3.5)$ as $L^{2}(\mathcal{M}) \otimes \mathrm{H}_{F}$ instead of $s p\left(L^{2}(\mathcal{M})\right) \otimes \mathcal{H}_{F}$. It is a remarkable "coincidence" that the passage from the standard model to the grand algebra, namely from $a=2$ to $a^{\prime}=4=2 a$, requires to multiply the dimension of the internal Hilbert space by 4 (for $2\left(2 a^{\prime}\right)^{2}=2(4 a)^{2}=4\left(2(2 a)^{2}\right)$ ) which is precisely the dimension of spinors in a spacetime of dimension 4 . Once more we stress that no new particles are introduced: $\mathcal{A}_{\mathcal{F}}$ acts on $\mathcal{H}_{F}=C^{32}, \mathcal{A}_{G}$ acts on $\mathrm{H}_{F}=C^{128}$ but $C^{\infty}(\mathcal{M}) \otimes \mathcal{A}_{G}$ and $C^{\infty}(\mathcal{M}) \otimes \mathcal{A}_{\mathcal{F}}$ acts on the same Hilbert space $\mathcal{H}$.

The representation of the grand algebra $\mathcal{A}_{G}$ on $\mathrm{H}_{F}$ is more involved than the one of $\mathcal{A}_{\mathcal{F}}$ on $\mathcal{H}_{F}$ in the previous section. In analogy with what was done earlier we consider an element of $\mathcal{A}_{G}$ as two $8 \times 8$ matrices, and see both of them having a block structure of four $4 \times 4$ matrices. Thus the component $Q \in M_{4}(H)$ of the grand algebra gets two

\footnotetext{
${ }^{8} \mathrm{D}, \mathrm{J}, \beta$, have the same range as $\mathrm{C}, \mathrm{I}, \alpha$ and serve as contracting indices.
} 
new extra indices with respect to the quaternionic component of $\mathcal{A}_{\mathcal{F}}$, and the same is true for $M \in M_{8}(C)$. For the quaternions we choose to identify these two new indices with the spinor (anti)-particles indices $\dot{0}, \dot{1}$; and for the complex matrices with the spinor left-right indices $r, l$ introduced in section 3 . This choice is not unique, and we leave a full investigation of the possible alternatives for future work. Having both sectors diagonal on different indices ensures that the order zero condition is satisfied, as explained below.

We therefore have

$$
Q=\left(\begin{array}{cc}
Q_{\dot{0} \alpha}^{\dot{0} \beta} & Q_{\dot{\phi} \alpha}^{\dot{1} \beta} \\
Q_{\dot{1} \alpha}^{0 \beta} & Q_{\dot{1} \alpha}^{1 \beta}
\end{array}\right)_{\dot{s} \dot{t}}^{1 \dot{m}} \in M_{4}(H), \quad M=\left(\begin{array}{cc}
M_{r \mathrm{~J}}^{r \mathrm{~J}} & M_{r \mathrm{~J}}^{l \mathrm{I}} \\
M_{l \mathrm{~J}}^{r \mathrm{I}} & M_{l \mathrm{~J}}^{l \mathrm{I}}
\end{array}\right)_{s t} \in M_{8}(C)
$$

where, for any $\dot{s}, \dot{t} \in\{\dot{0}, \dot{1}\}$ and $s, t \in\{l, r\}$, the matrices

$$
Q_{\dot{s} \alpha}^{\dot{t} \beta} \in M_{2}(H), \quad M_{s \mathrm{~J}}^{t \mathrm{I}} \in M_{4}(C)
$$

have the index structure defined below (3.8). This means that the representation of the element $A=(Q, M) \in \mathcal{A}_{G}$ is: ${ }^{9}$

$$
A_{\mathrm{D} s \mathrm{~J} s \alpha}^{\mathrm{C} t \dot{t} \beta}=\left(\delta_{0}^{\mathrm{C}} \delta_{s}^{t} \delta_{\mathrm{J}}^{\mathrm{I}} Q_{\dot{s} \alpha}^{\dot{t} \beta}+\delta_{1}^{\mathrm{C}} M_{s \mathrm{~J}}^{t \mathrm{I}} \delta_{\dot{s}}^{\dot{t}} \delta_{\alpha}^{\beta}\right)
$$

This representation is to be compared with (3.8). As before the quaternionic part acts on the particle sector of the internal indices $\left(\delta_{0}^{C}\right)$ and the complex part on the antiparticle sector $\left(\delta_{1}^{\mathrm{C}}\right)$. The difference is that the grand algebra acts in a nondiagonal way not only on the flavour and lepto-colour indices $\alpha, \mathrm{I}$, but also on the $s$ and $\dot{s}$ indices. The novelty is in this mixing of internal and spacetime indices: at the grand algebra level, the spin structure is somehow hidden. Specifically, the representation (3.12) is not invariant under the action of the Lorentz group (or rather of Spin(4) since we are dealing with spin representation, in Euclidean signature). This point is adressed in section 5.2.

The representation of $C^{\infty}(\mathcal{M}) \otimes \mathcal{A}_{G}$ is given by (3.12) where the entries of $Q$ and $M$ are now functions on $\mathcal{M}$. Since the total Hilbert space $\mathcal{H}$ is unchanged, there is not reason to change the real structure and the grading. In particular one easily checks that the order zero condition holds true for the grand algebra

$$
\left[A, J B J^{-1}\right]=0 \quad \forall A, B \in \mathcal{A}_{G} .
$$

This is because the real structure $J$ in (2.7) acts as the charge conjugation operator

$$
\mathcal{J}=i \gamma^{0} \gamma^{2} c c=i\left(\begin{array}{cc}
\bar{\sigma}_{\dot{s}}^{2} & 0_{2} \\
0_{2} & \sigma^{2}{ }_{\dot{s}}^{\dot{t}}
\end{array}\right)_{s t} c c
$$

on the spinor indices, and as $J_{F}$ in $\mathcal{H}_{F}$ (where it exchanges the two blocks corresponding to particles and antiparticles). In tensorial notations one has

$$
(J \Psi)_{s \dot{s} \alpha}^{\mathrm{CI}}=-i \eta_{s}^{t} \tau_{\dot{s}}^{\dot{t}} \xi_{\mathrm{D}}^{\mathrm{C}} \delta_{\mathrm{J}}^{\mathrm{I}} \delta_{\alpha}^{\beta} \bar{\Psi}_{t \dot{t} \beta}^{\mathrm{DJ}}
$$

\footnotetext{
${ }^{9}$ To take into account the non-diagonal action of $Q$ and $M$, it is convenient to change the order of the indices with respect to (3.8). We now adopt the order: $C, s, I, \dot{s}, \alpha$.
} 
where we use Einstein summation and define

$$
\xi=\left(\begin{array}{ll}
0 & 1 \\
1 & 0
\end{array}\right)_{\mathrm{CD}}, \quad \eta=\left(\begin{array}{cc}
1 & 0 \\
0 & -1
\end{array}\right)_{s t}, \quad \tau=\left(\begin{array}{cc}
0 & -1 \\
1 & 0
\end{array}\right)_{\dot{s} \dot{t}} .
$$

Hence $J$ preserves the indices structure in (3.12), apart from the exchange $\delta_{0}^{\mathrm{C}} \leftrightarrow \delta_{1}^{\mathrm{C}}$ : since $Q$ and $M$ act on different indices, the commutation (3.13) is assured. Notice that without the enlargement of the action of the finite dimensional algebra to the spinorial indices, it would have been impossible to find a representation of $\mathcal{A}_{G}$ which satisfies the order zero condition, unless one adds more fermions. In this respect the grand algebra is not anymore an internal algebra.

\subsection{Reduction due to grading}

In a way similar to the reduction $\mathcal{A}_{\mathcal{F}} \rightarrow \mathcal{A}_{\mathcal{L R}}$ explained in appendix $\mathrm{A}$, the grading condition imposes a reduction $\mathcal{A}_{G} \rightarrow \mathcal{A}_{G}^{\prime}$ where

$$
\mathcal{A}_{G}^{\prime}=\left(M_{2}(H)_{L} \oplus M_{2}(H)_{R}\right) \oplus\left(M_{4}(C)_{l} \oplus \mathbb{M}_{4}(C)_{r}\right) .
$$

To see it, recall that the chirality $\Gamma$ in $(2.7)$ acts as $\gamma^{5}=\eta_{s}^{t} \delta_{\dot{s}}^{t}$ on the spin indices, and as $\gamma_{F}$ on the internal indices:

$$
(\Gamma \Psi)_{s \dot{s} \alpha}^{\mathrm{CI}}=\eta_{s}^{t} \delta_{\dot{s}}^{\dot{t}} \eta_{\mathrm{D}}^{\mathrm{C}} \delta_{\mathrm{J}}^{\mathrm{I}} \eta_{\alpha}^{\beta} \Psi_{t \dot{t} \beta}^{\mathrm{DJ}}
$$

where $\eta_{\mathrm{D}}^{\mathrm{C}}$ and $\eta_{\alpha}^{\beta}$ are defined as in (3.16). Changing the order of the indices so that to match (3.12), one has

$$
\Gamma=\eta_{\mathrm{D}}^{\mathrm{C}} \eta_{s}^{t} \delta_{\mathrm{J}}^{\mathrm{I}} \delta_{\dot{s}}^{\dot{t}} \eta_{\alpha}^{\beta}
$$

Since the representation of $\mathcal{A}_{G}$ is diagonal in the $\mathrm{C}$ index, the grading condition is satisfied if and only if it is satisfied by both sectors - quaternionic and complex - independently.

For quaternions, one asks $\left[\eta_{s}^{t} \delta_{\mathrm{J}}^{\mathrm{I}} \delta_{\dot{s}}^{\dot{t}} \eta_{\alpha}^{\beta}, \delta_{s}^{t} \delta_{\mathrm{J}}^{\mathrm{I}} Q_{\dot{s} \alpha}^{\dot{t} \beta}\right]=0$, that is $\left[\delta_{\dot{s}}^{t} \eta_{\alpha}^{\beta}, Q_{\dot{s} \alpha}^{\dot{t} \beta}\right]=0$. This imposes

$$
Q=\left(\begin{array}{ll}
\mathrm{Q}_{\dot{0} \alpha}^{\dot{0} \beta} & \mathrm{Q}_{\dot{0} \alpha}^{\dot{1} \beta} \\
\mathrm{Q}_{\dot{1} \alpha}^{0 \beta} & \mathrm{Q}_{\dot{1} \alpha}^{1 \beta}
\end{array}\right)_{\dot{s} \dot{t}}
$$

where for any $\dot{s}, \dot{t} \in\{\dot{0}, \dot{1}\}$ one has

$$
\mathrm{Q}_{\dot{t} \alpha}^{\dot{s} \beta}=\left(\begin{array}{cc}
q_{R_{\dot{t}}}^{\dot{s}} & 0_{2} \\
0_{2} & q_{L_{\dot{t}}^{\dot{s}}}^{\dot{s}}
\end{array}\right)_{\alpha \beta} \text { with } \quad q_{R_{\dot{t}}}^{\dot{s}}, q_{L \dot{t}}^{\dot{s}} \in H .
$$

Elements of the kind (3.20) generates $M_{2}(H)_{R} \oplus M_{2}(H)_{L}$. Hence the reduction

$$
M_{4}(H) \rightarrow M_{2}(H)_{R} \oplus M_{2}(H)_{L}
$$

For matrices, one asks $\left[\eta_{s}^{t} \delta_{\mathrm{J}}^{\mathrm{I}} \delta_{\dot{s}}^{t} \eta_{\alpha}^{\beta}, M_{s \mathrm{~J}}^{t \mathrm{I}} \delta_{\dot{s}}^{\dot{t}} \delta_{\alpha}^{\beta}\right]=0$, that is $\left[\eta_{s}^{t} \delta_{\mathrm{J}}^{\mathrm{I}}, M_{s \mathrm{~J}}^{t \mathrm{I}}\right]=0$. This forces

$$
M=\left(\begin{array}{cc}
M_{r \mathrm{~J}}^{r \mathrm{I}} & 0_{4} \\
0_{4} & M_{l \mathrm{~J}}^{l \mathrm{I}}
\end{array}\right)_{s t}
$$


meaning the reduction

$$
M_{8}(C) \rightarrow M_{4}(C)_{r} \oplus M_{4}(C)_{l}
$$

Hence the reduction of the grand algebra to $\mathcal{A}_{G}^{\prime}$. Notice that the grading causes a reduction not only in the quaternionic sector, as in the case of $\mathcal{A}_{\mathcal{F}}$, but also in the complex matrix part. This is because $\mathcal{A}_{G}$ is not anymore acting only on internal indices.

\section{The Majorana coupling and the $\sigma$ field}

In this section we see how the grand algebra makes possible to have a Majorana mass giving rise to the field $\sigma$. Although the calculations are quite involved, the principle is quite simple. Since we have a larger algebra, the Majorana Dirac operator needs not be diagonal in the spin indices. This added degree of freedom enables the possibility to satisfy the order one condition in a non trivial way, namely to still have a one form which commutes with the opposite algebra, but that at the same time gives rise to a field. In the following we will show this analytically, all calculations have also been performed with a symbolic manipulation programme, leading to the same results.

We first work out in section 4.1 the most general Dirac operator $D_{\nu}$ with Majorana coupling compatible with the grading condition and the $K O$ dimension of the spectral triple of the standard model. Then we study the first order condition induced by $D_{\nu}$ and the subsequent reduction $\mathcal{A}_{G} \rightarrow \mathcal{A}_{G}^{\prime \prime}$ of the grand algebra (4.2). Finally we show in 4.3 that $D_{\nu}$ can be fluctuated by $\mathcal{A}_{G}^{\prime \prime}$ so that to generate the field $\sigma$ as required by (2.15).

\subsection{Dirac operator with Majorana mass term}

We will consider a Majorana-like mass only for the right handed neutrinos. This choice is dictated by physics, and elsewhere we will investigate the more general case. The natural mass scale of this matrix is very high, so that it provides a natural see-saw mechanism (although in realistic scheme the right handed neutrino mass is somewhat lower than the Planck scale). The standard model can be considered as a low energy limit of the theory we present in this section. We will assume therefore that all the quantities involved in the internal Dirac operator $D_{F}$ but the Majorana coupling are small compared to the scale of the breaking described here. Moreover we work with one generation only, meaning that $D_{F}=D_{R}$ is given by (B.1). We take advantage of the flexibility introduced by the grand algebra and we do not assume a priori that the Majorana coupling is diagonal on the spin indices. This means that instead of $\gamma^{5} \otimes D_{F}$ as in (2.9) we consider a finite dimensional matrix $D_{\nu}$ containing a Majorana mass term with non trivial action on the spin indices. Right handed neutrinos have indices $\mathrm{I}=0$ and $\alpha=1$, so that the most general Majorana coupling matrix is

$$
D_{\nu}=\mathcal{R} \otimes D_{R}=\left(\begin{array}{cc}
0_{64} & \mathrm{D}_{\nu} \\
\mathrm{D}_{\nu}^{\dagger} & 0_{64}
\end{array}\right)_{\mathrm{CD}} \quad \text { with } \quad \mathrm{D}_{\nu}=\mathcal{R}_{s \dot{s}}^{t \dot{t}} \quad \Xi_{\mathrm{J}}^{\mathrm{I}} \Xi_{\alpha}^{\beta}
$$


where $\mathcal{R}$ is - at this stage - an arbitrary $4 \times 4$ complex matrix while $\Xi$ is the projector on the first component

$$
\Xi=\left(\begin{array}{cc}
1 & 0 \\
0 & 0
\end{array}\right)
$$

The constraints on the matrix $\mathcal{R}$ come from the grading condition and the real structure. Remembering (3.19), one has that $\Xi_{\mathrm{J}}^{\mathrm{I}}$ and $\Xi_{\alpha}^{\beta}$ commute with $\delta_{\mathrm{J}}^{\mathrm{I}}$ and $\eta_{\alpha}^{\beta}$, while the r.h.s. of (4.1) as a matrix in CD anticommutes with $\eta_{\mathrm{D}}^{\mathrm{C}}$. So $D_{\nu}$ anticommutes with $\Gamma$ if and only if $\mathcal{R}$ commutes with $\gamma^{5}$, meaning that $\mathcal{R}$ is block diagonal

$$
\mathcal{R}=\left(\begin{array}{cc}
\mathcal{R}_{r \dot{s}}^{r \dot{t}} & 0_{2} \\
0_{2} & \mathcal{R}_{l \dot{s}}^{l i}
\end{array}\right)_{s t}=:\left(\begin{array}{cc}
\mathrm{r}_{\dot{s}}^{\dot{t}} & 0_{2} \\
0_{2} & \left.\right|_{\dot{s}} ^{\dot{t}}
\end{array}\right)_{s t}
$$

The requirement to have $K O$-dimension $2 \bmod 8$ means that $J D_{\nu}=D_{\nu} J$. Remembering (3.15), this is equivalent to

$$
\left[-i\left(\begin{array}{cc}
0_{4} & \eta_{s}^{t} \tau_{\dot{s}}^{\dot{t}} \\
\eta_{s}^{t} \tau_{\dot{s}}^{t} & 0_{4}
\end{array}\right)_{C D} c c,\left(\begin{array}{cc}
0_{4} & \mathcal{R}_{s \dot{s}}^{t \dot{t}} \\
\mathcal{R}_{s \dot{s}}^{\dagger t \dot{t}} & 0_{4}
\end{array}\right)_{C D}\right]=0
$$

that is

$$
(\tau \otimes \eta) \mathcal{R}^{T}-\mathcal{R}(\tau \otimes \eta)=0, \quad(\tau \otimes \eta) \overline{\mathcal{R}}-\mathcal{R}^{\dagger}(\tau \otimes \eta)=0
$$

By (4.3), the first equation above yields (omitting the $s t$ and $\dot{s} \dot{t}$ indices)

$$
\left(\begin{array}{cc}
\tau & 0_{2} \\
0_{2} & -\tau
\end{array}\right)\left(\begin{array}{cc}
r^{T} & 0_{2} \\
0_{2} & \mathrm{I}^{T}
\end{array}\right)-\left(\begin{array}{cc}
r & 0_{2} \\
0_{2} & \mathrm{l}
\end{array}\right)\left(\begin{array}{cc}
\tau & 0_{2} \\
0_{2} & -\tau
\end{array}\right)=0
$$

i.e. $\mathbf{r} \tau=\tau \mathbf{r}^{T}$ and $\mathbf{|} \tau=\left.\tau\right|^{T}$, whose solution is

$$
\mathrm{r}_{\dot{s}}^{i}=k_{r} \delta_{\dot{s}}^{l}, \mathrm{l}_{\dot{s}}^{l}=k_{l} \delta_{\dot{s}}^{l} \quad k_{r}, k_{l} \in C
$$

The second equation in (4.5) is then satisfied as well.

Eq. (4.7), (4.3) and (4.1) give the most general Dirac operator $D_{\nu}$ on $L^{2}\left(R^{4}\right) \otimes \mathrm{H}_{F}$, with Majorana mass term, coupling the right neutrino with its anti-particle. In tensorial notations, one has

$$
\mathrm{D}_{\nu}=\kappa_{s}^{t} \Xi_{\mathrm{J}}^{\mathrm{I}} \delta_{\dot{s}}^{\dot{t}} \Xi_{\alpha}^{\beta} \quad \text { where } \quad \kappa=\left(\begin{array}{cc}
k_{r} & 0 \\
0 & k_{l}
\end{array}\right)_{s t} .
$$

By choosing $k_{r}=-k_{l}=1$, one gets $\mathcal{R}=\gamma^{5}$ and one retrieves the Majorana coupling $D_{\nu}=\gamma^{5} \otimes D_{R}$ of the standard model. However, at this stage nothing forces us to make this choice. 


\subsection{First order condition for Majorana Dirac operator}

We aim at obtaining the field $\sigma$ as a fluctuation of $D_{\nu}$, compatible with the first order condition. By (3.12) a generic element $(Q, M)$ of $\mathcal{A}_{G}$ acts as ${ }^{10}$

$$
A=\left(\begin{array}{cc}
\delta_{s \mathrm{~J}}^{t \mathrm{I}} Q_{\dot{s} \alpha}^{\dot{t} \beta} & 0_{64} \\
0_{64} & M_{s \mathrm{~J}}^{t \mathrm{I}} \delta_{\dot{s} \beta}^{t \alpha}
\end{array}\right)_{\mathrm{CD}}=:\left(\begin{array}{cc}
\mathrm{Q} & 0_{64} \\
0_{64} & \mathrm{M}
\end{array}\right)_{\mathrm{CD}} .
$$

As well, with $B=(R, N) \in \mathcal{A}_{G}$, a generic element of the opposite algebra is

$$
J B J^{-1}=-J B J=-\left(\begin{array}{cc}
\tilde{N}_{s \mathrm{~J}}^{t \mathrm{I}} \delta_{\dot{s} \beta}^{t \alpha} & 0_{64} \\
0_{64} & \delta_{s \mathrm{~J}}^{t \mathrm{I}} \tilde{R}_{\dot{s} \beta}^{t \beta}
\end{array}\right)_{\mathrm{CD}}=-\left(\begin{array}{cc}
\tilde{\mathrm{N}} & 0_{64} \\
0_{64} & \tilde{\mathrm{R}}
\end{array}\right)_{\mathrm{CD}}
$$

where we define

$$
\tilde{R}_{\dot{s} \alpha}^{i \beta}=(\tau \bar{R} \tau)_{\dot{s} \alpha}^{i \beta}, \quad \tilde{N}_{s \mathrm{~J}}^{t \mathrm{I}}=-(\eta \bar{N} \eta)_{s \mathrm{~J}}^{t \mathrm{I}}=-\bar{N}_{s \mathrm{~J}}^{t \mathrm{I}} .
$$

The first order condition for $D_{\nu}$ means that

$$
\begin{aligned}
0 & =\left[\left[D_{\nu}, A\right], J B J^{-1}\right]=\left[\left[\left(\begin{array}{cc}
0_{64} & \mathrm{D}_{\nu} \\
\mathrm{D}_{\nu}^{\dagger} & 0_{64}
\end{array}\right)_{\mathrm{CD}},\left(\begin{array}{cc}
\mathrm{Q} & 0_{64} \\
0_{64} & \mathrm{M}
\end{array}\right)_{\mathrm{CD}}\right],\left(\begin{array}{cc}
\tilde{\mathrm{N}} & 0_{64} \\
0_{64} & \tilde{\mathrm{R}}
\end{array}\right)_{\mathrm{CD}}\right] \\
& =\left(\begin{array}{c}
0_{64} \\
\mathrm{D}_{\nu}^{\dagger} \mathrm{Q} \tilde{\mathrm{N}}-\mathrm{MD}_{\nu}^{\dagger} \tilde{\mathrm{N}}-\tilde{\mathrm{R}} \mathrm{D}_{\nu}^{\dagger} \mathrm{Q}+\tilde{\mathrm{R} M D_{\nu}^{\dagger}} \quad \mathrm{D}_{\nu} \mathrm{MR}-\mathrm{QD}_{\nu} \tilde{\mathrm{R}}-\tilde{\mathrm{N}} \mathrm{D}_{\nu} \mathrm{M}+\tilde{\mathrm{N}} \mathrm{QD}_{\nu}
\end{array}\right)_{\mathrm{CD}}
\end{aligned}
$$

We look for solutions that satisfy the grading condition, i.e. in $\mathcal{A}^{\prime}{ }_{G}$. Inspired by the first order condition for $\mathcal{A}_{L R}$ and $D_{F}$ described in appendix A, we also impose the reductions

$$
M_{4}(C)_{r} \rightarrow C_{r} \oplus M_{3}(C)_{r}, \quad M_{4}(C)_{l} \rightarrow C_{l} \oplus M_{3}(C)_{l}
$$

as well as

$$
M_{2}(H)_{R} \rightarrow H_{R} \oplus H_{R}^{\prime}, \quad M_{2}(H)_{L} \rightarrow H_{L} \oplus H_{L}^{\prime} .
$$

The reduction (4.13) is obtained assuming that the components in (3.23) are $(i, j=1,2,3)$

$$
\begin{aligned}
& M_{r \mathrm{~J}}^{r \mathrm{I}}=\left(\begin{array}{cc}
M_{r 0}^{r 0} & 0 \\
0 & M_{r j}^{r i}
\end{array}\right)_{\mathrm{IJ}}=:\left(\begin{array}{cc}
m_{r} & 0 \\
0 & M_{r j}^{r i}
\end{array}\right)_{\mathrm{IJ}} \quad m_{r} \in C_{r}, \\
& M_{l \mathrm{~J}}^{l \mathrm{I}}=\left(\begin{array}{cc}
M_{l 0}^{l 0} & 0 \\
0 & M_{l j}^{l i}
\end{array}\right)_{\mathrm{IJ}}=:\left(\begin{array}{cc}
m_{l} & 0 \\
0 & M_{l j}^{l i}
\end{array}\right)_{\mathrm{IJ}} m_{l} \in C_{l} .
\end{aligned}
$$

The reduction (4.14) is obtained imposing that the off-diagonal part of $Q$ in (3.20) is zero:

$$
Q=\left(\begin{array}{cc}
Q_{\dot{0} \alpha}^{\dot{\beta}} & 0_{4} \\
0_{4} & \mathrm{Q}_{\dot{1} \alpha}^{\dot{i} \beta}
\end{array}\right)_{\dot{s} \dot{t}}
$$

where

$$
\mathrm{Q}_{\dot{0} \alpha}^{\dot{\alpha} \beta}=\left(\begin{array}{cc}
q_{R} & 0_{2} \\
0_{2} & q_{L}
\end{array}\right)_{\alpha \beta}, \quad \mathrm{Q}_{\dot{1} \alpha}^{\dot{i} \beta}=\left(\begin{array}{cc}
q_{R}^{\prime} & 0_{2} \\
0_{2} & q_{L}^{\prime}
\end{array}\right)_{\alpha \beta} \quad q_{R, L} \in H_{R, L}, \quad q_{R, L}^{\prime} \in H_{R, L}^{\prime} .
$$

\footnotetext{
${ }^{10}$ To lighten notation, for any pairs of indices $x, y$ and $u, v$ we write $\delta_{y v}^{x u}=\delta_{y}^{x} \delta_{v}^{u}$.
} 
Finally we impose that $q_{R}$ and $q_{R}^{\prime}$ are diagonal quaternions, that is

$$
q_{R}=\left(\begin{array}{cc}
c_{R} & 0 \\
0 & \bar{c}_{R}
\end{array}\right)_{\dot{s} \dot{t}}, \quad q_{R}^{\prime}=\left(\begin{array}{cc}
c_{R}^{\prime} & 0 \\
0 & \bar{c}_{R}^{\prime}
\end{array}\right)_{\dot{s} \dot{t}} \quad \text { with } c_{R}, c_{R}^{\prime} \in C,
$$

meaning the reduction $H_{R} \oplus H_{R}^{\prime} \rightarrow C_{R} \oplus C_{R}^{\prime}$. We thus look for solutions of (4.12) in

$$
\mathcal{A}_{G}^{\prime \prime}=\left(H_{L} \oplus H_{L}^{\prime} \oplus C_{R} \oplus C_{R}^{\prime}\right) \oplus\left(C_{l} \oplus M_{3}(C)_{l} \oplus C_{r} \oplus M_{3}(C)_{r}\right) .
$$

Notice that we do not claim there is no solution of (4.12) outside $\mathcal{A}_{G}^{\prime \prime}$. But for our purposes, it turns out that it is sufficient to work with $\mathcal{A}_{G}^{\prime \prime}$.

Under these conditions, the first equation coming from (4.12), namely

$$
\mathrm{D}_{\nu} \mathrm{M} \tilde{\mathrm{R}}-\mathrm{QD}_{\nu} \tilde{\mathrm{R}}-\tilde{\mathrm{N}} \mathrm{D}_{\nu} \mathrm{M}+\tilde{\mathrm{N}} \mathrm{QD}_{\nu}=0
$$

has explicit components

$$
\begin{aligned}
& \mathrm{D}_{\nu} \mathrm{M} \tilde{\mathrm{R}}=\left(\kappa_{s}^{t} \Xi_{\mathrm{J}}^{\mathrm{I}} \delta_{\dot{s}}^{t} \Xi_{\alpha}^{\beta}\right)\left(M_{s \mathrm{~J}}^{t \mathrm{I}} \delta_{\dot{s} \beta}^{t \alpha}\right)\left(\delta_{s \mathrm{I}}^{t \mathrm{~J}} \tilde{R}_{\dot{s} \alpha}^{\dot{t} \beta}\right)=(\kappa \Xi M)_{s \mathrm{~J}}^{t \mathrm{I}}(\Xi \tilde{R})_{\dot{s} \alpha}^{\dot{i} \beta} \\
& =\left(\begin{array}{cc}
k_{r} \mathrm{~m}_{r} & 0_{4} \\
0_{4} & k_{l} \mathrm{~m}_{l}
\end{array}\right)_{s t} \otimes\left(\begin{array}{cc}
-\overline{\mathrm{d}}_{R}^{\prime} & 0_{4} \\
0_{4} & -\overline{\mathrm{d}}_{R}
\end{array}\right)_{\dot{s} \dot{t}} ; \\
& \mathrm{QD}_{\nu} \tilde{\mathrm{R}}=\left(\delta_{s \mathrm{I}}^{t \mathrm{~J}} Q_{\dot{s} \alpha}^{i \beta}\right)\left(\kappa_{s}^{t} \Xi_{\mathrm{J}}^{\mathrm{I}} \delta_{\dot{s}}^{\dot{t}} \Xi_{\alpha}^{\beta}\right)\left(\delta_{s \mathrm{I}}^{t \mathrm{~J}} \tilde{R}_{\dot{s} \alpha}^{i \beta}\right)=(\kappa \Xi)_{s \mathrm{~J}}^{t \mathrm{I}}(Q \Xi \tilde{R})_{\dot{s} \alpha}^{i \beta} \\
& =\left(\begin{array}{cc}
k_{r} \Xi & 0_{4} \\
0_{4} & k_{l} \Xi
\end{array}\right)_{s t} \otimes\left(\begin{array}{cc}
-\mathrm{c}_{R} \overline{\mathrm{d}}_{R}^{\prime} & 0_{4} \\
0_{4} & -\mathrm{c}_{R}^{\prime} \overline{\mathrm{d}}_{R}
\end{array}\right)_{s \dot{s}} ; \\
& \tilde{\mathrm{N}} \mathrm{D}_{\nu} \mathrm{M}=\left(\tilde{\mathrm{N}}_{s \mathrm{~J}}^{t \mathrm{I}} \delta_{\dot{s} \alpha}^{t \beta}\right)\left(\kappa_{s}^{t} \Xi_{\mathrm{J}}^{\mathrm{I}} \delta_{\dot{s}}^{t} \Xi_{\alpha}^{\beta}\right)\left(\mathrm{M}_{s \mathrm{~J}}^{t \mathrm{I}} \delta_{\dot{s} \alpha}^{t \beta}\right)=\left((\tilde{\mathrm{N}} \kappa \Xi M)_{s \mathrm{~J}}^{t \mathrm{I}}(\delta \Xi)_{\dot{s} \alpha}^{i \beta}=\right. \\
& =\left(\begin{array}{cc}
-k_{r} \overline{\mathrm{n}_{r}} \mathrm{~m}_{r} & 0_{4} \\
0_{4} & -k_{l} \overline{\mathrm{n}_{l}} \mathrm{~m}_{l}
\end{array}\right)_{s t} \otimes\left(\begin{array}{cc}
\Xi & 0_{4} \\
0_{4} & \Xi
\end{array}\right)_{\dot{s} \dot{t}} ; \\
& \tilde{\mathrm{N}} \mathrm{QD}_{\nu}=\left(\tilde{\mathrm{N}}_{s \mathrm{~J}}^{t \mathrm{I}} \delta_{\dot{s} \beta}^{t \alpha}\right)\left(\delta_{s \mathrm{I}}^{t \mathrm{~J}} \mathrm{Q}_{\dot{s} \alpha}^{i \beta}\right)\left(\kappa_{s}^{t} \Xi_{\mathrm{J}}^{\mathrm{I}} \delta_{\dot{s}}^{\dot{t}} \Xi_{\alpha}^{\beta}\right)=(\tilde{\mathrm{N}} \kappa \Xi)_{s \mathrm{~J}}^{t \mathrm{I}}(\mathrm{Q} \Xi)_{\dot{s} \alpha}^{i \beta}= \\
& =\left(\begin{array}{cc}
-k_{r} \overline{n_{r}} & 0_{4} \\
0_{4} & -k_{l} \overline{\mathrm{n}_{l}}
\end{array}\right)_{s t} \otimes\left(\begin{array}{cc}
c_{R} & 0_{4} \\
0_{4} & c_{R}^{\prime}
\end{array}\right)_{\dot{s} \dot{t}}
\end{aligned}
$$

where we defined the $4 \times 4$ complex matrices

$$
\mathrm{m}_{r, l}=\left(\begin{array}{cc}
m_{r, l} & 0 \\
0 & 0_{3}
\end{array}\right)_{\mathrm{IJ}} \quad \mathrm{c}_{R, L}=\left(\begin{array}{cc}
c_{R, L} & 0 \\
0 & 0_{3}
\end{array}\right)_{\alpha \beta} \quad \mathrm{c}_{R, L}^{\prime}=\left(\begin{array}{cc}
c_{R, L}^{\prime} & 0 \\
0 & 0_{3}
\end{array}\right)_{\alpha \beta}
$$

with $m_{r}, m_{l}$ the components of $M$ and $c_{R}, c_{R}^{\prime}$ the one of $Q$. Similarly we define the matrices $\mathrm{n}_{r, l}$ from the components $n_{l, r}$ of $N$, and the matrices $\mathrm{d}, \mathrm{d}_{R}^{\prime}$ from the components $d_{R}, d_{R}^{\prime}$ of R. The matrix $\Xi$ carries the indices I, J in the second equation, and $\alpha, \beta$ in the third. In each equation, to pass from the first to the second lines one uses (4.11).

Collecting the components and assuming that both $k_{r}$ and $k_{l}$ are non zero, one finds that (4.20) is equivalent to

$$
\begin{gathered}
\left(c_{R}-m_{r}\right)\left(\bar{n}_{r}-\bar{d}_{R}^{\prime}\right)=0, \quad\left(\bar{d}_{R}-\bar{n}_{r}\right)\left(m_{r}-c_{R}^{\prime}\right)=0 \\
\left(c_{R}-m_{l}\right)\left(\bar{n}_{l}-\bar{d}_{R}^{\prime}\right)=0, \quad\left(\bar{d}_{R}-\bar{n}_{l}\right)\left(m_{l}-c_{R}^{\prime}\right)=0 .
\end{gathered}
$$


A similar calculation for the second components of (4.12) yields the same system of equations. Thus one solution to the first order condition induced by $D_{\nu}$ is to impose

$$
c_{R}=m_{r}=m_{l} \text { and } d_{R}=n_{r}=n_{l},
$$

meaning the reduction of $\mathcal{A}_{G}^{\prime \prime}$ to

$$
\mathcal{A}_{G}^{\prime \prime \prime}=H_{L} \oplus H_{L}^{\prime} \oplus C_{R}^{\prime} \oplus C \oplus M_{3}(C)_{l} \oplus M_{3}(C)_{r}
$$

\subsection{The $\sigma$ field as a 1 -form}

We now consider the set of 1 -forms $\sum_{i} B_{i}\left[D_{\nu}, A_{i}\right]$ generated by the Majorana Dirac operator and the algebra $\mathcal{A}_{G}^{\prime \prime \prime}$ above. We are interested in showing that this set is non empty, and it is enough to consider the simplest 1 -form

$$
\left[D_{\nu}, A\right]=\left(\begin{array}{cc}
0_{64} & \mathrm{D}_{\nu} \mathrm{M}-\mathrm{QD}_{\nu} \\
\mathrm{D}_{\nu}^{\dagger} \mathrm{Q}-\mathrm{MD}_{\nu}^{\dagger} & 0_{64}
\end{array}\right)
$$

We begin with $A=(Q, M)$ in $\mathcal{A}_{G}^{\prime}$. With notations of the precedent section, one has

$$
\begin{aligned}
& \mathrm{D}_{\nu} \mathrm{M}-\mathrm{QD}_{\nu}=\left(\kappa_{s}^{t} \Xi_{\mathrm{J}}^{\mathrm{I}} \delta_{\dot{s}}^{t} \Xi_{\alpha}^{\beta}\right)\left(M_{s \mathrm{~J}}^{t \mathrm{I}} \delta_{\dot{s} \alpha}^{t \beta}\right)-\left(\delta_{s \mathrm{I}}^{t \mathrm{~J}} Q_{\dot{s} \alpha}^{t \beta}\right)\left(\kappa_{s}^{t} \Xi_{\mathrm{J}}^{\mathrm{I}} \delta_{\dot{s}}^{t} \Xi_{\alpha}^{\beta}\right) \\
& =(\kappa \Xi M)_{s \mathrm{~J}}^{t \mathrm{I}}(\Xi \delta)_{\dot{s} \alpha}^{i \beta}-(\kappa \Xi)_{s \mathrm{I}}^{t \mathrm{~J}}(Q \Xi)_{\dot{s} \alpha}^{i \beta} \\
& =\left(\begin{array}{cc}
k_{r} \mathrm{~m}_{r} & 0_{4} \\
0_{4} & k_{l} \mathrm{~m}_{l}
\end{array}\right)_{s t} \otimes\left(\begin{array}{cc}
\Xi & 0_{4} \\
0_{4} & \Xi
\end{array}\right)_{\dot{s} \dot{t}}-\left(\begin{array}{cc}
k_{r} \Xi & 0_{4} \\
0_{4} & k_{l} \Xi
\end{array}\right)_{s t} \otimes\left(\begin{array}{cc}
\mathrm{c}_{R} & 0_{4} \\
0_{4} & \mathrm{c}_{R}^{\prime}
\end{array}\right)_{\dot{s} \dot{t}}
\end{aligned}
$$

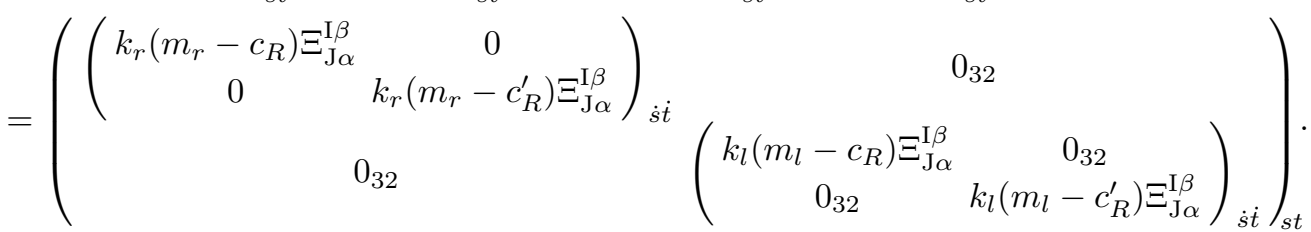

By the reduction $\mathcal{A}^{\prime}{ }_{G} \rightarrow \mathcal{A}^{\prime \prime \prime}{ }_{G}$, the component $k_{r}\left(m_{r}-c_{R}\right)$ vanishes, but the component $k_{l}\left(m_{l}-c_{R}^{\prime}\right)$ does not. This is the crucial difference with the algebra of the standard model: the grand algebra allows to generates a non-vanishing 1-form associated to the Majorana Dirac operator $D_{\nu}$, which satisfies the first order condition.

Restoring the order $s \dot{s} \mathrm{I} \alpha$ of the indices, the matrix above is $\mathcal{R}=\mathcal{R}_{s \dot{s}}^{t \dot{t}} \Xi_{\mathrm{I}}^{\mathrm{J}} \Xi_{\alpha}^{\beta}$ with

$$
\mathcal{R}_{s \dot{s}}^{t \dot{t}}=\left(\begin{array}{cc}
\left(\begin{array}{cc}
0 & 0 \\
0 & k_{r}\left(m_{r}-c_{R}^{\prime}\right)
\end{array}\right)_{\dot{s} \dot{t}} & 0_{2} \\
0_{2} & \left(\begin{array}{cc}
0 & 0 \\
0 & k_{l}\left(m_{r}-c_{R}^{\prime}\right)
\end{array}\right)_{\dot{s} \dot{s}}
\end{array}\right)_{s t} .
$$

For anti-selfadjoint $A$ (that is $\mathrm{M}=-\mathrm{M}^{\dagger}, \mathrm{Q}=-\mathrm{Q}^{\dagger}$ ), one obtains the selfadjoint 1-form

$$
\left[D_{\nu}, A\right]=\left(\begin{array}{cc}
0_{64} & \mathcal{R} \\
\mathcal{R}^{\dagger} & 0_{64}
\end{array}\right) .
$$


The conjugate action of the real structure $J$ yields

$$
J\left[D_{\nu}, A\right] J^{-1}=-J\left[D_{\nu}, A\right] J=-\left(\begin{array}{cc}
0_{64} & \mathcal{J} \mathcal{R}^{\dagger} \mathcal{J} \\
\mathcal{J} \mathcal{R} \mathcal{J} & 0_{64}
\end{array}\right)
$$

where the charge conjugation $\mathcal{J}$ acts only on the spin indices. Explicitly, omitting the factor $\Xi_{\mathrm{I}}^{\mathrm{J}} \Xi_{\alpha}^{\beta}$ in the expression of $\mathcal{R}$, one gets

$$
\begin{aligned}
& \mathcal{J} \mathcal{R}^{\dagger} \mathcal{J}=\eta_{s}^{t} \tau_{\dot{s}}^{\dot{t}}\left(\mathcal{R}^{T}\right)_{s \dot{s}}^{t \dot{t}} \eta_{s}^{t} \tau_{\dot{s}}^{\dot{t}}=\left(\begin{array}{ccc}
\tau_{\dot{s}}^{\dot{t}} \mathcal{R}_{r \dot{s}}^{r \dot{t}} \tau_{\dot{s}}^{\dot{t}} & 0_{4} \\
0_{4} & \tau_{\dot{s}}^{\dot{t}} \mathcal{R}_{l \dot{s}}^{l \dot{t}} \tau_{\dot{s}}^{\dot{t}}
\end{array}\right)_{s t} \\
& \left.=-\left(\begin{array}{cc}
\left(k_{r}\left(m_{r}-c_{R}^{\prime}\right)\right. & 0 \\
0 & 0
\end{array}\right)_{\dot{s} \dot{t}}\left(\begin{array}{cc}
k_{l}\left(m_{r}-c_{R}^{\prime}\right) & 0 \\
0 & 0
\end{array}\right)_{\dot{s} \dot{t}}\right)_{s t} \text {, }
\end{aligned}
$$

that is $-\mathcal{J R}^{\dagger} \mathcal{J}$ is obtained by permuting the components in the blocks $\dot{s} \dot{t}$ of $\mathcal{R}$. As well

$$
\mathcal{J} \mathcal{R} \mathcal{J}=\eta_{s}^{t} \tau_{\dot{s}}^{\dot{t}} \overline{\mathcal{R}}_{s \dot{s}}^{t \dot{t}} \eta_{s}^{t} \tau_{\dot{s}}^{\dot{t}}=\eta_{s}^{t} \tau_{\dot{s}}^{\dot{t}}\left(\mathcal{R}^{\dagger}\right)_{s \dot{s}}^{t \dot{t}} \eta_{s}^{t} \tau_{\dot{s}}^{\dot{t}}
$$

is obtained from $-\mathcal{R}^{\dagger}$ by permuting the components in $\dot{s} \dot{t}$. Consequently,

$$
D_{\nu}+\left[D_{\nu}, A\right]+J\left[D_{\nu}, A\right] J^{-1}=\left(\begin{array}{cc}
0_{64} & \mathcal{M}_{\nu} \\
\mathcal{M}_{\nu}^{\dagger} & 0_{64}
\end{array}\right)
$$

where $\mathcal{M}_{\nu}=\mathrm{R}_{s \dot{s}}^{t \dot{t}} \Xi_{\mathrm{I}}^{\mathrm{J}} \Xi_{\alpha}^{\beta}$ with

$$
\mathrm{R}=\left(\begin{array}{cc}
k_{r}\left(1+\left(m_{r}-c_{R}^{\prime}\right)\right) \delta_{\dot{s}}^{\dot{t}} & 0_{2} \\
0_{2} & k_{l}\left(1+\left(m_{r}-c_{R}^{\prime}\right)\right) \delta_{\dot{s}}^{\dot{t}}
\end{array}\right)_{s t} .
$$

Now, considering that $A$ is in $C^{\infty}(\mathcal{M}) \otimes \mathcal{A}_{G}^{\prime \prime}$, the coefficients $m_{r}$ and $c_{R}^{\prime}$ becomes functions on the manifold $\mathcal{M}$. Taking $k_{l}=-k_{r}=k_{R}$, one obtains $\mathrm{R}_{s \dot{s}}^{t \dot{t}}=k_{R} \sigma \gamma^{5}$ where

$$
\sigma=\left(1+\left(m_{r}-c_{R}^{\prime}\right)\right)
$$

is now a field on $\mathcal{M}$. In other terms, the fluctuation of $D_{\nu}$ by $\mathcal{A}_{G}$ yields the substitution (2.15). The grand algebra gives a justification for the presence of the field $\sigma$, necessary to obtain the mass of the Higgs in agreement with experiment.

\section{Reduction to the standard model}

Starting with the grand algebra $\mathcal{A}_{G}$ reduced to $\mathcal{A}_{G}^{\prime}$ by the grading condition, we have shown how to generate the field $\sigma$ by a fluctuation of the Majorana-Dirac operator $D_{\nu}$, in a way satisfying the first order condition imposed by $D_{\nu}$. As explained below (4.8), one can choose in particular $D_{\nu}=\gamma^{5} \otimes D_{R}$, where $D_{R}$ is the internal Dirac operator $D_{F}$ of the standard model in which only the dominant term (i.e. the Majorana mass) is taken into account. In other words, the field $\sigma$ is generated by fluctuating the second term in the Dirac operator (2.9) of the standard model. We now show that the first order condition of the first term in (2.9), i.e. the free Dirac operator, yields the reduction of the grand algebra to the standard model. 


\subsection{First order condition for the free Dirac operator}

The first term in (2.9) is the Euclidean free Dirac operator, extended trivially to the internal space of one generation. In tensorial notation it reads

$$
\not \partial \otimes \mathbb{I}_{32}=-i \delta_{\mathrm{DJ} \alpha}^{\mathrm{CI} \beta} \gamma^{\mu} \partial_{\mu} .
$$

For $A=(Q, M) \in C^{\infty}(\mathcal{M}) \otimes \mathcal{A}_{G}^{\prime}$, the commutator

$$
\left[\not \partial \otimes I_{32}, A\right]=-i\left(\begin{array}{cc}
\delta_{\mathrm{J}}^{\mathrm{I}}\left[\gamma^{\mu} \partial_{\mu} \delta_{\alpha}^{\beta}, \delta_{s}^{t} Q_{\dot{s} \alpha}^{\dot{\epsilon} \beta}\right] & 0_{64} \\
0_{64} & {\left[\gamma^{\mu} \partial_{\mu} \delta_{\mathrm{J}}^{\mathrm{I}}, M_{s \mathrm{~J}}^{t \mathrm{I}}\right] \delta_{\alpha}^{\beta}}
\end{array}\right)_{\mathrm{CD}}
$$

has components (omitting the non relevant $\delta$ )

$$
\begin{aligned}
{\left[\gamma^{\mu} \partial_{\mu} \delta_{\alpha}^{\beta}, \delta_{s}^{t} Q_{\dot{s} \alpha}^{\dot{t} \beta}\right] } & =\left[\left(\begin{array}{cc}
0_{8} & \sigma_{\dot{s}}^{\mu \dot{t}} \partial_{\mu} \delta_{\alpha}^{\beta} \\
\bar{\sigma}_{\dot{s}}^{\mu \dot{t}} \partial_{\mu} \delta_{\alpha}^{\beta} & 0_{8}
\end{array}\right)_{s t},\left(\begin{array}{cc}
Q_{\dot{s} \alpha}^{\dot{t} \beta} & 0_{8} \\
0_{8} & Q_{\dot{s} \alpha}^{\dot{t} \beta}
\end{array}\right)_{s t}\right] \\
& =\left(\begin{array}{cc}
0_{8} & P_{\dot{s} \alpha}^{\dot{t} \beta}+T_{\dot{s} \alpha}^{\dot{t} \beta, \mu} \partial_{\mu} \\
\bar{P}_{\dot{s} \alpha}^{\dot{t} \beta}+\bar{T}_{\dot{s} \alpha}^{\dot{t} \beta, \mu} \partial_{\mu} & 0_{8}
\end{array}\right)_{s t}
\end{aligned}
$$

where

$$
P_{\dot{s} \alpha}^{\dot{t} \beta}=\left(\sigma_{\dot{s}}^{\mu \dot{u}} \partial_{\mu} Q_{\dot{u} \alpha}^{\dot{t} \beta}\right), \quad T_{\dot{s} \alpha}^{\dot{t} \beta, \mu}=\left[\sigma_{\dot{s}}^{\mu \dot{t}}, Q_{\dot{s} \alpha}^{\dot{t} \beta}\right]
$$

and similar definitions for $\bar{P}$ and $\bar{T}$ with $\bar{\sigma}$ instead of $\sigma$; and

$$
\begin{aligned}
{\left[\gamma^{\mu} \partial_{\mu} \delta_{\mathrm{J}}^{\mathrm{I}}, M_{s \mathrm{~J}}^{t \mathrm{I}} \delta_{\dot{s}}^{\dot{t}}\right] } & =\left[\left(\begin{array}{cc}
0_{8} & \sigma_{\dot{s}}^{\mu \dot{t}} \partial_{\mu} \delta_{\mathrm{J}}^{\mathrm{I}} \\
\bar{\sigma}_{\dot{s}}^{\mu \dot{t}} \partial_{\mu} \delta_{\mathrm{J}}^{\mathrm{I}} & 0_{8}
\end{array}\right)_{s t},\left(\begin{array}{cc}
M_{r \mathrm{~J}}^{r \mathrm{I}} \delta_{\dot{s}}^{\dot{t}} & 0_{8} \\
0_{8} & M_{l \mathrm{~J}}^{l \mathrm{I}} \delta_{\dot{s}}^{\dot{t}}
\end{array}\right)_{s t}\right] \\
& =\left(\begin{array}{cc}
0_{8} & L_{\mathrm{J} \dot{s}}^{\mathrm{I} \dot{t}}+K_{\mathrm{J} \dot{s}}^{\mathrm{I} \dot{t}, \mu} \partial_{\mu} \\
\bar{L}_{\mathrm{J} \dot{s}}^{\mathrm{I} \dot{t}}+\bar{K}_{\mathrm{J} \dot{s}}^{\mathrm{I} \dot{t}, \mu} \partial_{\mu} & 0_{8}
\end{array}\right)_{s t}
\end{aligned}
$$

where

$$
\begin{array}{ll}
L_{\mathrm{J} \dot{s}}^{\mathrm{I} \dot{t}}=\left(\sigma_{\dot{s}}^{\mu \dot{t}} \partial_{\mu} M_{l \mathrm{~J}}^{l \mathrm{I}}\right), & K_{\mathrm{J} \dot{s}}^{\mathrm{I} \dot{t}, \mu}=\left(M_{l \mathrm{~J}}^{l \mathrm{I}}-M_{r \mathrm{~J}}^{r \mathrm{I}}\right) \sigma_{\dot{s}}^{\mu \dot{t}}, \\
\bar{L}_{\mathrm{J} \dot{s}}^{\mathrm{I} \dot{t}}=\left(\bar{\sigma}_{\dot{s}}^{\mu \dot{t}} \partial_{\mu} M_{r \mathrm{~J}}^{r \mathrm{I}}\right), & \bar{K}_{\mathrm{J} \dot{s}}^{\mathrm{I} \dot{t}, \mu}=\left(M_{r \mathrm{~J}}^{r \mathrm{I}}-M_{l \mathrm{~J}}^{l \mathrm{I}}\right) \bar{\sigma}_{\dot{s}}^{\mu \dot{t}} .
\end{array}
$$

For $B=(R, N) \in \mathcal{A}_{G}^{\prime}$, the commutator of $[\not \partial, A]$ with $J B J$ given in (4.10) is a block diagonal matrix in $\mathrm{CD}$ with components

$$
\begin{aligned}
& {\left[\delta_{\mathrm{J}}^{\mathrm{I}}\left[\gamma^{\mu} \partial_{\mu} \delta_{\alpha}^{\beta}, \delta_{s}^{t} Q_{\dot{s} \alpha}^{t \beta}\right], \tilde{N}_{s \mathrm{~J}}^{t \mathrm{I}} \delta_{\dot{s} \alpha}^{i \beta}\right]=} \\
& =\left[\left(\begin{array}{cc}
0_{32} & \delta_{\mathrm{J}}^{\mathrm{I}}\left(P_{\dot{s} \alpha}^{\dot{t} \beta}+T_{\dot{s} \alpha}^{\dot{t} \beta, \mu} \partial_{\mu}\right) \\
\delta_{\mathrm{J}}^{\mathrm{I}}\left(\bar{P}_{\dot{s} \alpha}^{\dot{t} \beta}+\bar{T}_{\dot{s} \alpha}^{\dot{t} \beta, \mu} \partial_{\mu}\right) & 0_{32}
\end{array}\right)_{s t},\left(\begin{array}{cc}
\tilde{N}_{r \mathrm{~J}}^{r \mathrm{I}} \delta_{\dot{s} \alpha}^{t \beta} & 0_{32} \\
0_{32} & \tilde{N}_{l \mathrm{~J}}^{l \mathrm{I}} \delta_{\dot{s} \alpha}^{i \beta}
\end{array}\right)_{s t}\right] ; \\
& {\left[\left[\gamma^{\mu} \partial_{\mu} \delta_{\mathrm{J}}^{\mathrm{I}}, M_{s \mathrm{~J}}^{t \mathrm{I}} \delta_{\dot{s}}^{\dot{t}}\right] \delta_{\alpha}^{\beta}, \delta_{s \mathrm{~J}}^{t \mathrm{I}} \tilde{R}_{\dot{s} \alpha}^{t \dot{\beta}}\right]=} \\
& =\left[\left(\begin{array}{cc}
0_{32} & \left(L_{\mathrm{J} \dot{s}}^{\mathrm{I} \dot{t}}+K_{\mathrm{J} \dot{s}}^{\mathrm{I} \dot{t}, \mu} \partial_{\mu}\right) \delta_{\alpha}^{\beta} \\
\left(\bar{L}_{\mathrm{J} \dot{s}}^{\mathrm{It}}+\bar{K}_{\mathrm{J} \dot{s}}^{\mathrm{It}, \mu} \partial_{\mu}\right) \delta_{\alpha}^{\beta} & 0_{32}
\end{array}\right)_{s t},\left(\begin{array}{cc}
\delta_{\mathrm{I}}^{\mathrm{J}} \tilde{R}_{\dot{s} \alpha}^{\dot{t} \beta} & 0_{32} \\
0_{32} & \delta_{\mathrm{I}}^{\mathrm{J}} \tilde{R}_{\dot{s} \alpha}^{i \beta}
\end{array}\right)_{s t}\right] .
\end{aligned}
$$


Omitting the indices (and noticing that the $P, T, \bar{P}, \bar{T}$ all commute with $\tilde{N}_{r}^{r}, \tilde{N}_{l}^{l}$ ), the first components is a diagonal matrix with first entry

$$
\left(\tilde{N}_{l}^{l}-\tilde{N}_{r}^{r}\right)\left(P+T^{\mu} \partial_{\mu}\right)+T^{\mu}\left(\partial_{\mu} \tilde{N}_{l}^{l}\right) .
$$

The vanishing of the differential operator part implies either $T^{\mu}=0$ or $\tilde{N}_{l}^{l}=\tilde{N}_{r}^{r}$. But the expression should be zero in particular for non-constant fields, that is for $P \neq 0$. So in case one imposes $T^{\mu}=0$, the vanishing of the term in $P$ implies $\tilde{N}^{l}=\tilde{N}^{r}$. In case one imposes $\tilde{N}_{l}^{l}=\tilde{N}_{r}^{r}$, the vanishing of the remaining term implies either $T^{\mu}=0$, or $\tilde{N}_{l}^{l}=\tilde{N}_{r}^{r}=$ cst. The last solution is unacceptable, it would mean that spacetime is reduced to a point, hence in any case one has both conditions: $T^{\mu}=0$ and $\tilde{N}_{l}^{l}=\tilde{N}_{r}^{r}$. One then checks that the other components of (5.7) vanish as well.

The only matrix that commutes with all the Pauli matrices is the identity, therefore

$$
T^{\mu}=0 \forall \mu \Longleftrightarrow Q_{\dot{0} \alpha}^{\dot{0} \beta}=Q_{\dot{1} \alpha}^{\dot{i} \beta} \text { and } Q_{\dot{1} \alpha}^{\dot{0} \beta}=Q_{\dot{0} \alpha}^{\dot{i} \beta}=0,
$$

meaning the breaking

$$
M_{2}(H)_{L} \oplus M_{2}(H)_{R} \rightarrow H_{L} \oplus H_{R}
$$

Meanwhile $\tilde{N}_{l}^{l}=\tilde{N}_{r}^{r}$ means that

$$
M_{4}(C)_{l} \oplus M_{4}(C)_{r} \rightarrow \mathbb{M}_{4}(C)
$$

Thus

$$
\mathcal{A}_{G}^{\prime} \rightarrow H_{L} \oplus H_{R} \oplus M_{4}(C)
$$

where representation of the r.h.s. algebra is now diagonal on the spinorial indices $\dot{s}, s$.

To summarize, the grand algebra $\mathcal{A}_{G}$ is broken by the chirality and the first order condition of the free Dirac operator to the left-right symmetric algebra $\mathcal{A}_{L R}$ of the standard model.

\subsection{Emergence of spin}

In noncommutative geometry the topological information is encoded in the algebra, while the geometry (e.g. the metric ${ }^{11}$ ) is in the $D$ operator. In particular the Riemann-spin structure is encoded in the way this operator, which contains the gamma matrices, acts on the Hilbert space. Without this operator there is just an algebra which acts in an highly reducible way on a 128 dimensional Hilbert space. This is conceptually what distinguishes $\mathcal{H}_{F}$ from $\mathrm{H}_{F}$ in (3.5): on $C^{\infty}(\mathcal{M}) \otimes \mathcal{H}_{F}$, the free Dirac operator (trivially extended to the internal indices) is

$$
\not \partial=-i \gamma^{\mu} \partial_{\mu} \otimes \delta_{\mathrm{DJ} \alpha}^{\mathrm{CI} \beta}
$$

On $C^{\infty}(\mathcal{M}) \otimes \mathrm{H}_{F}$ the same operator writes

$$
\not \partial=-i \partial_{\mu} \otimes \delta_{\mathrm{DJ} \alpha}^{\mathrm{CI} \beta} \gamma^{\mu}
$$

\footnotetext{
${ }^{11}$ The metric aspects of the almost commutative geometry of the standard model have been investigated in $[15,56]$
} 
and the spin structure, carried by the $\gamma$ matrices, is hidden among the internal degrees of freedom. In this sense the first order condition, which governs the passage from (5.14) to (5.13), corresponds to the emergence of the spin structure.

Alternatively, a spin structure means that the vectors in the Hilbert space transform in a particular representation under the "Lorentz" group. Since we are dealing with spinors in the Euclidean case, the group is actually $\operatorname{Spin}(4)$. It is generated by the commutators of the Dirac matrices, that act on $\mathcal{H}$ as

$$
S^{\mu \nu}:=\left[\gamma^{\mu}, \gamma^{\nu}\right] \otimes \mathbb{I}_{32}^{(\mathrm{CI} \alpha)} \text {. }
$$

Let us distinguish between an element $a$ of $C^{\infty}(\mathcal{M}) \otimes \mathcal{A}_{G}$ and its representation $\pi(a):=A$ given in (3.12). For any $\Lambda=\lambda_{\mu \nu} S^{\mu \nu} \in \operatorname{Spin}(4)$ and $A \in \pi\left(C^{\infty}(\mathcal{M}) \otimes \mathcal{A}_{G}\right)$, let

$$
\alpha_{\Lambda} A=\mathrm{U}(\Lambda) \operatorname{AU}(\Lambda)^{*} .
$$

The representation (3.12) of the grand algebra is not invariant under the adjoint action (5.16) of $\operatorname{Spin}(4)$ since $\alpha_{\Lambda} \pi(a)$ is not in $\pi\left(C^{\infty}(\mathcal{M}) \otimes \mathcal{A}_{G}\right)$. In this sense the representation of the grand algebra is not Lorentz invariant, unlike its reduction to $\mathcal{A}_{L R}$ which is diagonal in the spin indices. However, at the abstract level the algebra is preserved under Lorentz transformations since the latter are implemented by unitary operators: for any $\Lambda$ one has that $\alpha_{\Lambda}\left(\pi\left(C^{\infty}(\mathcal{M}) \otimes \mathcal{A}_{G}\right)\right)$ is isomorphic to $C^{\infty}(\mathcal{M}) \otimes \mathcal{A}_{G}$. This suggests to view the grand algebra as a phase of the universe in which the spin and rotation structure of spacetime has not yet emerged, only the topology (i.e. the abstract algebra) is fixed.

\subsection{Fiat neutrino}

The grand algebra together with the Majorana Dirac operator $D_{\nu}$ generates the field $\sigma$ at the right position (i.e. as required in (2.15)), respecting the first order condition induced by $D \nu$. However, by (4.35) one has that $\sigma$ becomes constant when one takes into account the first order condition imposed by the free Dirac operator, because (5.9) implies that $c_{R}^{\prime}=c_{R}=m_{r}$. This suggests a scenario in which the neutrino Majorana mass is the first field to appear and fluctuate, before the geometric structure of spacetime emerges through the breaking described in section 5.2. In this picture, the field $\sigma$ is viewed as a fluctuation of a vacuum that satisfies the first order condition of the free Dirac operator.

This scenario is supported by some preliminary calculations, which indicate that the first order condition of the free Dirac operator can be equivalently obtained as a minimum of the spectral action. In this way, the geometrical breaking imposed by the mathematical requirement of the theory becomes a dynamical breaking, and the field $\sigma$ would appear as the "Higgs field" associated to it. This idea has been investigated, in the case of the standard model algebra, in the recent papers [30,31]. The case of the grand algebra is in progress.

\section{Conclusions and outlook}

It is known that, although the spectral action requires the unification of interactions at a single scale, the usual grand unified theories, such as $\mathrm{SU}(5)$ or $\mathrm{SO}(10)$, do not fit in the 
noncommutative geometry framework, and are possible only renouncing to associativity [57, 58]. In this paper we pointed out there is a "next level" in noncommutative geometry, but that it is intertwined with the Riemannian and spin structure of spacetime, and therefore it naturally appears at a high scale. The added degrees of freedom are related to the Riemann-spin structure of spacetime, which emerges as a symmetry breaking very similar in nature to the Higgs mechanism. In addition, the higher symmetry explains the presence of the $\sigma$ field necessary for a correct fit of the mass of the Higgs. The results presented here, as is common in this model, are crucially depending on the Euclidean structure of the theory. This is particularly important as far as the role of chirality and the doubling of the degrees of freedom is concerned. A Wick rotation is far from simple in this context, and the construction of a Minkowskian noncommutative geometry is yet to come (for recent works see $[59,60])$.

The presence of this grand symmetry will have also phenomenological consequences which should be investigated. The breaking mechanisms we described in this paper are just barely sketched, we only looked at the group structure. A more punctual analysis should reveal more structure, and possibly alter the running of the constants at high energy.

For the moment we can only speculate. One of the problems of the spectral action in its present form is that it requires unification of the three gauge couplings at a single scale, $\Lambda$, and physical predictions are based on the value of this scale. It is known experimentally that in the absence of new physics the three constants do not meet in a single point, but the three lines form an elongated triangle spanning nearly three orders of magnitude. On the other side in the spectral action is not clear what would happen after this point, if one consider scales higher that $\Lambda$, i.e. earlier epochs. For a theory dealing with the unification of gauge theory and gravity a more natural scale is the Planck scale. An unification of the coupling constants at the Planck scale in the form of a pole has been considered [61, 62], but it requires new fermions. In the case at hand the "new physics" is in the form of a different structure which mixes spacetime spin and gauge degrees of freedom. This might have consequences for the interactions, and hence for the running of the various quantities, as well.

Other mathematical issues should be investigated. In particular the choice of the action of the grand algebra on the spin indices reflects how much the algebra is not diagonal in the "interaction" encoded by the free Dirac operator. Hopefully this could be interpreted at the light of Connes unitary invariant in Riemannian geometry [63].

\section{Acknowledgments}

We would like to thank the referee for careful readings of the manuscript, and for useful comments which improved the paper. We would like to thank M.A. Kurkov and D. Vassilevich for discussions. F. Lizzi acknowledges support by CUR Generalitat de Catalunya under project FPA2010-20807. P.M. warmly thanks A. Roche for constant support. 


\section{A Reduction of the $\mathcal{A}_{\mathcal{F}}$ algebra}

We give the details of the reduction of $\mathcal{A}_{\mathcal{F}}$ to $\mathcal{A}_{s m}$ by the grading and the first order condition. Rather than $\mathcal{H}_{R} \oplus \mathcal{H}_{L} \oplus \mathcal{H}_{R}^{c} \oplus \mathcal{H}_{L}^{c}$, it is convenient to work in the CI $\alpha$ basis of $\mathcal{H}_{F}=C^{32}$ (one generation of leptons $l$ and quarks $q$ ), namely

$$
\underbrace{\underbrace{\mathcal{H}_{l R} \oplus \mathcal{H}_{l L}}_{\mathrm{I}=0 ; \alpha=1, \ldots, 4} \oplus \underbrace{\left(\mathcal{H}_{q R} \oplus \mathcal{H}_{q L}\right) \otimes I_{3}}_{\mathrm{I}=i ; \alpha=1, \ldots, 4}}_{\mathrm{C}=0} \oplus \underbrace{\mathcal{H}_{l R}^{c} \oplus \mathcal{H}_{l L}^{c}}_{\mathrm{C}=1} \oplus \underbrace{\left(\mathcal{H}_{q R}^{c} \oplus \mathcal{H}_{q L}^{c}\right) \otimes I_{3}}_{\mathrm{I}=0 ; \alpha=1, \ldots, \alpha=1, \ldots, 4} .
$$

In this basis the internal Dirac operator is

$$
D_{F}=\left(\begin{array}{cc}
D_{1}^{1} & D_{2}^{1} \\
D_{1}^{2}=D_{2}^{1 \dagger} & D_{2}^{2}=\bar{D}_{1}^{1}
\end{array}\right)_{\mathrm{CD}}
$$

where

$$
D_{1}^{1}=\left(\begin{array}{cc}
\mathcal{M}^{0} & 0 \\
0 & \mathcal{M}^{i}
\end{array}\right)_{\mathrm{IJ}}, \quad D_{2}^{1}=\left(\begin{array}{cc}
\mathcal{M}^{R} & 0 \\
0 & 0 \\
& 0
\end{array}\right)_{\mathrm{IJ}}
$$

are $16 \times 16$ matrix with

$$
\mathcal{M}^{0}=\left(\begin{array}{cccc}
0 & 0 & \bar{k}_{\nu} & 0 \\
0 & 0 & 0 & \bar{k}_{e} \\
k_{\nu} & 0 & 0 & 0 \\
0 & k_{e} & 0 & 0
\end{array}\right)_{\alpha \beta}, \quad \mathcal{M}^{i=1,2,3}=\left(\begin{array}{cccc}
0 & 0 & \bar{k}_{u} & 0 \\
0 & 0 & 0 & \bar{k}_{d} \\
k_{u} & 0 & 0 & 0 \\
0 & k_{d} & 0 & 0
\end{array}\right)_{\alpha \beta}, \quad \mathcal{M}^{R}=\left(\begin{array}{cccc}
k_{R} & 0 & 0 & 0 \\
0 & 0 & 0 & 0 \\
0 & 0 & 0 & 0 \\
0 & 0 & 0 & 0
\end{array}\right)_{\alpha \beta}
$$

where $k_{e}, k_{u}, k_{d}, k_{\nu}$ are the Yukawa couplings of the electrons, quarks and neutrino, and $k_{R}$ is the neutrino Majorana mass.

Let us first examine the grading condition. By (3.8) the element $A=(Q, M) \in \mathcal{A}_{\mathcal{F}}$ act on $\mathcal{H}_{F}$ as

$$
A=\left(\begin{array}{cc}
\mathrm{Q} & 0_{16} \\
0_{16} & \mathrm{M}
\end{array}\right)_{\mathrm{CD}}
$$

where

$$
\mathrm{Q}=\delta_{\mathrm{J}}^{\mathrm{I}} Q_{\alpha}^{\beta} \in M_{2}(H), \quad \mathrm{M}=M_{\mathrm{J}}^{\mathrm{I}} \delta_{\alpha}^{\beta} \in M_{4}(C) .
$$

To guarantee that $A$ commutes with $\Gamma$, the factor $\eta_{\alpha}^{\beta}$ in (3.18) requires $Q$ to be diagonal in the $\alpha$ index, which reduces this part of the algebra to $\mathbb{H}_{L} \oplus \mathbb{H}_{R}$. This means that $Q_{\beta}^{\alpha}$ in (3.8) acts separately on the left and right handed doublets. No such breaking occurs in complex part, because $\delta_{\mathrm{J}}^{\mathrm{I}}$ in (3.18) does not put any constraints on $M_{4}(C)$. Likewise, $\eta_{s}^{t}$ does not produce any breaking because the action of both quaternions and complex matrices is diagonal on the spin indices. Thus we have $\mathcal{A}_{\mathcal{F}} \rightarrow \mathcal{A}_{L R}$.

Let us now examine the first order condition. For any $B=(R, N) \in \mathcal{A}_{L R}$ one has

$$
J_{F} B J_{F}=\left(\begin{array}{cc}
\overline{\mathrm{N}} & 0_{16} \\
0_{16} & \overline{\mathrm{R}}
\end{array}\right)_{\mathrm{CD}}
$$


Assuming there is no neutrino Majorana mass (i.e $D_{2}^{1}=0$ ), the first order condition for the finite dimensional spectral triple yields

$$
\left[\left[D_{F}, A\right], J_{F} B J_{F}\right]=\left(\begin{array}{c}
{\left[\left[D_{1}^{1}, \mathrm{Q}\right], \overline{\mathrm{N}}\right]} \\
0_{16}
\end{array}\left[\begin{array}{c}
0_{16} \\
\left.\left.\bar{D}_{1}^{1}, \mathrm{M}\right], \overline{\mathrm{R}}\right]
\end{array}\right)_{\mathrm{CD}}=0\right.
$$

Let $n_{\mathrm{J}}^{\mathrm{I}}$ be the components of $\bar{N} \in M_{4}(C)$. The upper-left term in the r.h.s. of (A.8) is

$$
\left[\left(\begin{array}{cc}
{\left[\mathcal{M}^{0}, Q\right]_{\alpha}^{\beta}} & 0 \\
0 & {\left[\mathcal{M}^{i}, Q\right]_{\alpha}^{\beta} \otimes I_{3}}
\end{array}\right)_{I J},\left(\begin{array}{cc}
n_{0}^{0} \delta_{\alpha}^{\beta} & n_{j}^{0} \delta_{\alpha}^{\beta} \\
n_{0}^{i} \delta_{\alpha}^{\beta} & n_{0}^{i} \delta_{\alpha}^{\beta}
\end{array}\right)_{I J}\right] .
$$

It is zero if and only if

$$
n_{i}^{0}\left[\mathcal{M}^{0}-\mathcal{M}^{i}, Q\right]_{\alpha}^{\beta}=n_{0}^{i}\left[\mathcal{M}^{0}-\mathcal{M}^{i}, Q\right]_{\alpha}^{\beta}=0 \quad \forall i=1,2,3 .
$$

Writing $Q=\left(\begin{array}{cc}q_{R} & 0 \\ 0 & q_{L}\end{array}\right) \in H_{L} \oplus H_{R}$ with $q_{R}=\left(\begin{array}{cc}a_{1} & a_{2} \\ -\bar{a}_{2} & \bar{a}_{1}\end{array}\right)$ and $q_{L}=\left(\begin{array}{cc}a_{3} & a_{4} \\ -\bar{a}_{4} & \bar{a}_{3}\end{array}\right)$ this means

$$
\begin{aligned}
n_{i}^{0}\left(a_{1}-a_{3}\right)\left(k_{\nu}-k_{u}\right)=n_{i}^{0}\left(a_{1}-a_{3}\right)\left(k_{e}-k_{d}\right) & =0, i=1,2,3 \\
n_{i}^{0}\left(a_{2}\left(k_{\nu}-k_{u}\right)-a_{4}\left(k_{e}-k_{d}\right)\right)=n_{i}^{0}\left(a_{2}\left(k_{e}-k_{d}\right)-a_{4}\left(k_{\nu}-k_{u}\right)\right) & =0, i=1,2,3
\end{aligned}
$$

and a similar equation for $n_{0}^{i}$.

A first solution could be $a_{1}=a_{3}$ and $a_{2}=a_{4}=0$, which means that the quaternionic part reduces to $C$ while $M_{4}(C)$ is not touched. The gauge group is then $\mathrm{U}(1) \times \mathrm{U}(4)$, which is to small to contain the gauge group of the standard model. The other solution is imposing $n_{0}^{i}=n_{i}^{0}=0$, which yields the reduction $\mathbb{M}_{4}(\mathbb{C}) \rightarrow \mathbb{C} \oplus \mathbb{M}_{3}(\mathbb{C})$. Then the second component of (A.8) vanishes as well. Thus the first order condition without Majorana mass, together with the grading condition, induces the breaking:

$$
\mathcal{A}_{\mathcal{F}} \rightarrow \mathcal{A}_{L R} \rightarrow\left(H_{L} \oplus H_{R}\right) \oplus\left(C \oplus M_{3}(C)\right) .
$$

A non-zero Majorana coupling $k_{R}$ (a constant at this stage) in the Dirac operator induces new terms in (A.8):

$$
\begin{aligned}
& {\left[\left[\left(\begin{array}{cc}
0_{16} & D_{2}^{1} \\
D_{2}^{1 \dagger} & 0_{16}
\end{array}\right),\left(\begin{array}{cc}
\mathrm{Q} & 0_{16} \\
0_{16} & \mathrm{M}
\end{array}\right)\right],\left(\begin{array}{cc}
\overline{\mathrm{N}} & 0_{16} \\
0_{16} & \overline{\mathrm{R}}
\end{array}\right)\right] } \\
= & \left(\begin{array}{c}
0_{16} \\
D_{2}^{1^{\dagger}} \mathrm{Q} \overline{\mathrm{N}}-\mathrm{M} D_{2}^{1 \dagger} \overline{\mathrm{N}}-\overline{\mathrm{R}} D_{2}^{1 \dagger} \mathrm{Q}+\overline{\mathrm{R} M}{D_{2}^{1 \dagger}}_{D_{2}^{1} \mathrm{MR}-\mathrm{Q} D_{2}^{1} \overline{\mathrm{R}}-\overline{\mathrm{N}} D_{2}^{1} \mathrm{M}+\overline{\mathrm{N}} \mathrm{Q} D_{2}^{1}}^{0_{16}}
\end{array}\right) .
\end{aligned}
$$

Writing $\overline{\mathrm{R}}=\left(\begin{array}{cc}q_{R}^{\prime} & 0 \\ 0 & q_{L}^{\prime}\end{array}\right) \in H_{L} \oplus H_{R}$ with $q_{R}^{\prime}=\left(\begin{array}{cc}b_{1} & b_{2} \\ -\bar{b}_{2} & \bar{b}_{1}\end{array}\right), q_{L}^{\prime}=\left(\begin{array}{cc}b_{3} & b_{4} \\ -\bar{b}_{4} & \bar{b}_{3}\end{array}\right)$, one gets

$$
\begin{aligned}
& D_{2}^{1} \mathrm{M} \overline{\mathrm{R}}=\left(\begin{array}{cc}
\left(\mathcal{M}^{R} \bar{R}\right)_{\alpha}^{\beta} & 0 \\
0 & 0_{12}
\end{array}\right)_{I J}\left(\begin{array}{cc}
m_{0}^{0} \delta_{\alpha}^{\beta} & 0 \\
0 & m_{j}^{i} \delta_{\alpha}^{\beta}
\end{array}\right)_{I J}
\end{aligned}
$$

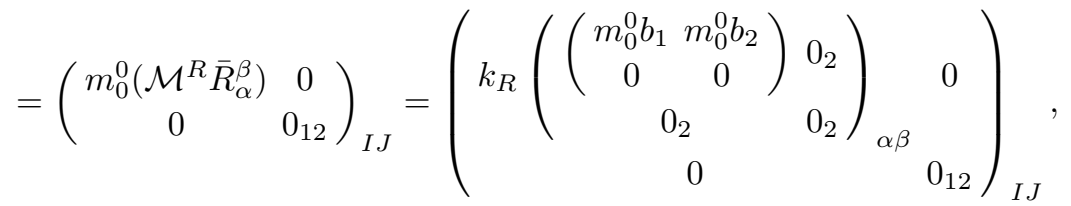


and similarly

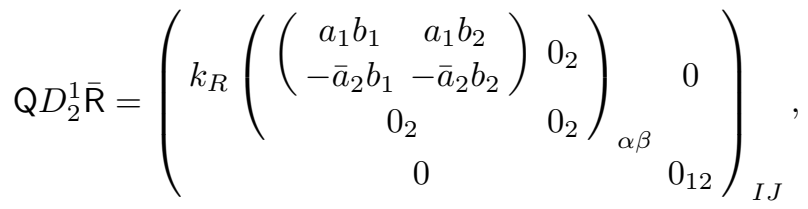

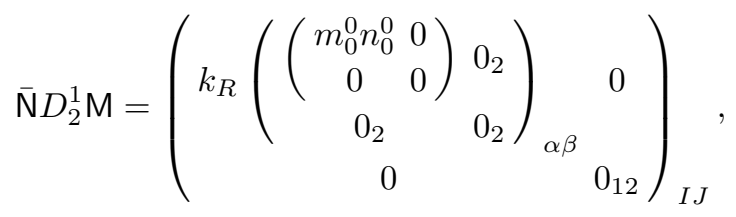

$$
\begin{aligned}
& \overline{\mathrm{N}} \mathrm{Q} D_{2}^{1}=\left(\begin{array}{ccc}
k_{R}\left(\left(\begin{array}{cc}
n_{0}^{0} a_{1} & 0 \\
-n_{0}^{0} \bar{a}_{2} & 0
\end{array}\right)\right. & 0_{2} \\
0_{2} & & \\
0 & & 0 \\
0 & & 0_{12}
\end{array}\right)_{I J}
\end{aligned}
$$

Asking (A.14) to be zero is thus equivalent to the system

$$
\begin{aligned}
m_{0}^{0} b_{1}-a_{1} b_{1}-m_{0}^{0} n_{0}^{0}+n_{0}^{0} a_{1} & =\left(m_{0}^{0}-a_{1}\right)\left(b_{1}-n_{0}^{0}\right)=0 \\
b_{2}\left(m_{0}^{0}-a_{1}\right) & =0, \quad \bar{a}_{2} b_{1}-n_{0}^{0} \bar{a}_{2}=0, \quad \bar{a}_{2} b_{2}=0,
\end{aligned}
$$

leading to $a_{1}=m_{0}^{0}, b_{1}=n_{0}^{0}$ and $a_{2}=b_{2}=0$. This means

$$
\left(\mathbb{H}_{L} \oplus \mathbb{H}_{R}\right) \oplus\left(\mathbb{C} \oplus \mathbb{M}_{3}(\mathbb{C})\right) \rightarrow\left(\mathbb{H}_{L} \oplus \mathbb{C}^{\prime}\right) \oplus\left(\mathbb{C} \oplus \mathbb{M}_{3}(\mathbb{C})\right)
$$

with

$$
\mathbb{C}=\mathbb{C}^{\prime}
$$

Hence the standard model algebra $\mathcal{A}_{s m}=\mathbb{C} \oplus \mathbb{H} \oplus \mathbb{M}_{3}(\mathbb{C})$.

\section{B Fluctuation of $D_{R}$ by the standard model algebra}

The notations are the one of appendix A. One easily checks that the set of 1 -forms induced by the Majorana Dirac operator

$$
D_{R}=\left(\begin{array}{cc}
0_{16} & D_{2}^{1} \\
D_{2}^{1 \dagger} & 0_{16}
\end{array}\right)_{\mathrm{CD}}
$$

is actually zero. Indeed, an element of $\mathcal{A}_{s m}$ is

$$
A=(\mathrm{Q}, \mathrm{M}) \quad \text { with } \quad \mathrm{Q}=\delta_{\mathrm{J}}^{\mathrm{I}} Q_{\alpha}^{\beta}, \quad \mathrm{M}=M_{\mathrm{J}}^{\mathrm{I}} \delta_{\alpha}^{\beta}
$$

where $Q_{\alpha}^{\beta}$ is as below (A.10) with $a_{2}=0$, and $M$ has components $m_{0}^{i}=m_{i}^{0}=0, i=1,2,3$. One thus gets

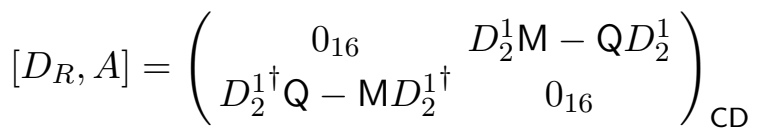


with

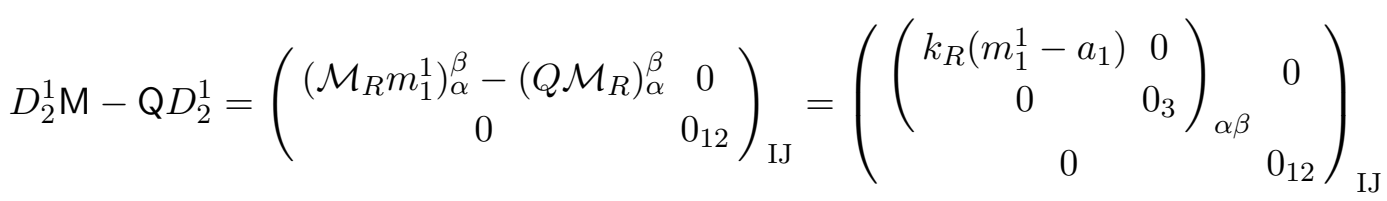

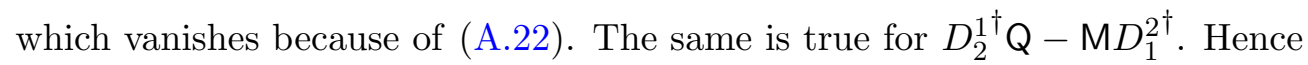

$$
\left[D_{R}, A\right]=0
$$

One may think of inverting the order of the reductions: first impose the first order condition of the Majorana-Dirac operator $D_{R}$, then the one of $D_{0}:=D_{F}-D_{R}$. By repeating the computation (A.14)-(A.18) with $A, B$ elements of $\mathcal{A}_{L R}$ (instead of being elements of the algebra at the r.h.s. of (A.12)), one obtains extra-constraints

$$
\begin{aligned}
b_{1} m_{i}^{0}-m_{0}^{0} n_{i}^{0} & =0 \\
-m_{0}^{i} n_{0}^{0}+n_{0}^{i} a_{1} & =0 \\
m_{0}^{i} n_{i}^{0} & =0
\end{aligned}
$$

whose solution is $m_{0}^{i}=m_{i}^{0}=0$. This means that the breaking

$$
\mathcal{A}_{L R} \rightarrow \mathcal{A}_{\text {sm }}
$$

can also be obtained directly from $D_{R}$ alone, without reducing first to the algebra (A.12).

Consequently, starting from $C^{\infty}(M) \otimes \mathcal{A}_{\mathcal{F}}$ reduced to $C^{\infty}(M) \otimes \mathcal{A}_{L R}$ by the grading condition, there is no way to fluctuate the Dirac operator - respecting the first order condition — so that to obtain the field $\sigma$ as required by eq. (2.15).

Open Access. This article is distributed under the terms of the Creative Commons Attribution License (CC-BY 4.0), which permits any use, distribution and reproduction in any medium, provided the original author(s) and source are credited.

\section{References}

[1] A. Connes, Noncommutative Geometry, Academic Press, 1984.

[2] G. Landi, An Introduction to noncommutative spaces and their geometry, in Springer Lecture Notes in Physics 51, Springer Verlag (Berlin Heidelberg), 1997, hep-th/9701078 [INSPIRE].

[3] J.M. Gracia-Bondia, J.C. Varilly and H. Figueroa, Elements of Noncommutative Geometry, Birkhauser, 2000.

[4] A. Connes and M. Marcolli, Noncommutative Geometry, Quantum Fields and Motives, American Mathematical Society, 2007.

[5] A. Connes and J. Lott, Particle Models and Noncommutative Geometry (Expanded Version), Nucl. Phys. Proc. Suppl. 18B (1991) 29 [InSPIRE].

[6] M. Dubois-Violette, J. Madore and R. Kerner, Classical Bosons in a Noncommutative Geometry, Class. Quant. Grav. 6 (1989) 1709 [INSPIRE]. 
[7] M. Dubois-Violette, R. Kerner and J. Madore, Noncommutative Differential Geometry of Matrix Algebras, J. Math. Phys. 31 (1990) 316 [InSPIRE].

[8] T. Schucker, Forces from Connes' geometry, Lect. Notes Phys. 659 (2005) 285 [hep-th/0111236] [INSPIRE].

[9] A.H. Chamseddine, A. Connes and M. Marcolli, Gravity and the standard model with neutrino mixing, Adv. Theor. Math. Phys. 11 (2007) 991 [hep-th/0610241] [INSPIRE].

[10] K. van den Dungen and W.D. van Suijlekom, Particle Physics from Almost Commutative Spacetimes, Rev. Math. Phys. 24 (2012) 1230004 [arXiv: 1204.0328] [InSPIRE].

[11] A. Connes, On the spectral characterization of manifolds, arXiv:0810.2088 [INSPIRE].

[12] A. Connes, Gravity coupled with matter and foundation of noncommutative geometry, Commun. Math. Phys. 182 (1996) 155 [hep-th/9603053] [INSPIRE].

[13] A.H. Chamseddine and A. Connes, Why the Standard Model, J. Geom. Phys. 58 (2008) 38 [arXiv:0706.3688] [INSPIRE].

[14] A.H. Chamseddine and A. Connes, Noncommutative Geometry as a Framework for Unification of all Fundamental Interactions including Gravity. Part I, Fortsch. Phys. 58 (2010) 553 [arXiv: 1004.0464] [inSPIRE].

[15] A.H. Chamseddine and A. Connes, The Spectral action principle, Commun. Math. Phys. 186 (1997) 731 [hep-th/9606001] [INSPIRE].

[16] K. Fujikawa, Comment on Chiral and Conformal Anomalies, Phys. Rev. Lett. 44 (1980) 1733 [INSPIRE].

[17] A.A. Andrianov and L. Bonora, Finite - Mode Regularization of the Fermion Functional Integral, Nucl. Phys. B 233 (1984) 232 [InSPIRE].

[18] A.A. Andrianov and L. Bonora, Finite Mode Regularization of the Fermion Functional Integral. 2., Nucl. Phys. B 233 (1984) 247 [INSPIRE].

[19] J. Polchinski, Renormalization and Effective Lagrangians, Nucl. Phys. B 231 (1984) 269 [INSPIRE].

[20] F. Lizzi and P. Vitale, Gauge and Poincaré' Invariant Regularization and Hopf Symmetries, Mod. Phys. Lett. A 27 (2012) 1250097 [arXiv:1202.1190] [inSPIRE].

[21] J.W. Barrett, A Lorentzian version of the non-commutative geometry of the standard model of particle physics, J. Math. Phys. 48 (2007) 012303 [hep-th/0608221] [INSPIRE].

[22] A.H. Chamseddine and A. Connes, Resilience of the Spectral Standard Model, JHEP 09 (2012) 104 [arXiv:1208.1030] [inSPIRE].

[23] J. Elias-Miro, J.R. Espinosa, G.F. Giudice, H.M. Lee and A. Strumia, Stabilization of the Electroweak Vacuum by a Scalar Threshold Effect, JHEP 06 (2012) 031 [arXiv:1203.0237] [INSPIRE].

[24] N. Krasnikov, Restriction of the Fermion Mass in Gauge Theories of Weak and Electromagnetic Interactions, Yad. Fiz. 28 (1978) 549 [INSPIRE].

[25] P.Q. Hung, Vacuum Instability and New Constraints on Fermion Masses, Phys. Rev. Lett. 42 (1979) 873 [INSPIRE].

[26] H.D. Politzer and S. Wolfram, Bounds on Particle Masses in the Weinberg-Salam Model, Phys. Lett. B 82 (1979) 242 [Erratum ibid. 83B (1979) 421] [INSPIRE]. 
[27] C.A. Stephan, New Scalar Fields in Noncommutative Geometry, Phys. Rev. D 79 (2009) 065013 [arXiv: 0901.4676] [INSPIRE].

[28] C.A. Stephan, Almost-commutative geometries beyond the standard model, J. Phys. A 39 (2006) 9657 [hep-th/0509213] [INSPIRE].

[29] C.A. Stephan, Almost-commutative geometries beyond the standard model. II. New Colours, J. Phys. A 40 (2007) 9941 [arXiv:0706.0595] [InSPIRE].

[30] A.H. Chamseddine, A. Connes and W.D. van Suijlekom, Inner Fluctuations in Noncommutative Geometry without the first order condition, J. Geom. Phys. 73 (2013) 222 [arXiv: 1304.7583] [INSPIRE].

[31] A.H. Chamseddine, A. Connes and W.D. van Suijlekom, Beyond the Spectral Standard Model: Emergence of Pati-Salam Unification, JHEP 11 (2013) 132 [arXiv:1304.8050] [INSPIRE].

[32] F. Lizzi, G. Mangano, G. Miele and G. Sparano, Fermion Hilbert space and fermion doubling in the noncommutative geometry approach to gauge theories, Phys. Rev. D 55 (1997) 6357 [hep-th/9610035] [INSPIRE].

[33] J.M. Gracia-Bondia, B. Iochum and T. Schucker, The Standard model in noncommutative geometry and fermion doubling, Phys. Lett. B 416 (1998) 123 [hep-th/9709145] [InSPIRE].

[34] F. Lizzi, G. Mangano, G. Miele and G. Sparano, Mirror fermions in noncommutative geometry, Mod. Phys. Lett. A 13 (1998) 231 [hep-th/9704184] [INSPIRE].

[35] P. Martinetti, Carnot-Caratheodory metric and gauge fluctuation in noncommutative geometry, Commun. Math. Phys. 265 (2006) 585 [hep-th/0506147] [INSPIRE].

[36] M.A. Rieffel, Morita equivalence for operator algebras, in Operator Algebras and Applications, R.V. Kadison ed., Proc. Symp. Pure Math. 285 (1982) 38, Amer. Math. Soc., Providence, 1982.

[37] P. Gilkey, Invariance Theory, the Heat Equation and the Athiya-Singer Index Theorem, Publish or Perish, 1984.

[38] D. Vassilevich, Heat kernel expansion: User's manual, Phys. Rept. 388 (2003) 279 [hep-th/0306138] [INSPIRE].

[39] A. Andrianov and F. Lizzi, Bosonic Spectral Action Induced from Anomaly Cancelation, JHEP 05 (2010) 057 [arXiv: 1001.2036] [INSPIRE].

[40] A. Andrianov, M. Kurkov and F. Lizzi, Spectral action, Weyl anomaly and the Higgs-Dilaton potential, JHEP 10 (2011) 001 [arXiv:1106.3263] [INSPIRE].

[41] M. Kurkov and F. Lizzi, Higgs-Dilaton Lagrangian from Spectral Regularization, Mod. Phys. Lett. A 27 (2012) 1250203 [arXiv:1210.2663] [INSPIRE].

[42] T. van den Broek and W.D. van Suijlekom, Supersymmetric QCD from noncommutative geometry, Phys. Lett. B 699 (2011) 119 [InSPIRE].

[43] W. Nelson and M. Sakellariadou, Natural inflation mechanism in asymptotic noncommutative geometry, Phys. Lett. B 680 (2009) 263 [arXiv:0903.1520] [INSPIRE].

[44] M. Sakellariadou, Cosmological consequences of the noncommutative spectral geometry as an approach to unification, J. Phys. Conf. Ser. 283 (2011) 012031 [arXiv:1010.4518] [INSPIRE].

[45] M. Marcolli and E. Pierpaoli, Early Universe models from Noncommutative Geometry, Adv. Theor. Math. Phys. 14 (2010) [arXiv:0908.3683] [INSPIRE]. 
[46] ATLAS collaboration, Observation of a new particle in the search for the Standard Model Higgs boson with the ATLAS detector at the LHC, Phys. Lett. B 716 (2012) 1 [arXiv: 1207.7214] [INSPIRE].

[47] CMS collaboration, Observation of a new boson at a mass of $125 \mathrm{GeV}$ with the CMS experiment at the LHC, Phys. Lett. B 716 (2012) 30 [arXiv:1207.7235] [INSPIRE].

[48] I. Pris and T. Schucker, Noncommutative geometry beyond the standard model, J. Math. Phys. 38 (1997) 2255 [hep-th/9604115] [INSPIRE].

[49] M. Paschke, F. Scheck and A. Sitarz, Can (noncommutative) geometry accommodate leptoquarks?, Phys. Rev. D 59 (1999) 035003 [hep-th/9709009] [INSPIRE].

[50] T. Schucker and S. Zouzou, Spectral action beyond the standard model, hep-th/0109124 [INSPIRE].

[51] R. Squellari and C.A. Stephan, Almost-Commutative Geometries Beyond the Standard Model. III. Vector Doublets, J. Phys. A 40 (2007) 10685 [arXiv:0706.3112] [InSPIRE].

[52] C.A. Stephan, Beyond the Standard Model: A Noncommutative Approach, arXiv:0905.0997 [INSPIRE].

[53] A.H. Chamseddine and A. Connes, Conceptual Explanation for the Algebra in the Noncommutative Approach to the Standard Model, Phys. Rev. Lett. 99 (2007) 191601 [arXiv: 0706.3690] [INSPIRE].

[54] J.C. Pati and A. Salam, Lepton Number as the Fourth Color, Phys. Rev. D 10 (1974) 275 [Erratum ibid. D 11 (1975) 703] [INSPIRE].

[55] F. Lizzi, G. Mangano, G. Miele and G. Sparano, Constraints on unified gauge theories from noncommutative geometry, Mod. Phys. Lett. A 11 (1996) 2561 [hep-th/9603095] [INSPIRE].

[56] P. Martinetti and R. Wulkenhaar, Discrete Kaluza-Klein from scalar fluctuations in noncommutative geometry, J. Math. Phys. 43 (2002) 182 [hep-th/0104108] [INSPIRE].

[57] R. Wulkenhaar, The Standard model within nonassociative geometry, Phys. Lett. B 390 (1997) 119 [hep-th/9607096] [INSPIRE].

[58] S. Farnsworth and L. Boyle, Non-Associative Geometry and the Spectral Action Principle, arXiv:1303.1782 [INSPIRE].

[59] M. Paschke and R. Verch, Local covariant quantum field theory over spectral geometries, Class. Quant. Grav. 21 (2004) 5299 [gr-qc/0405057] [inSPIRE].

[60] N. Franco, Lorentzian approach to noncommutative geometry, arXiv:1108.0592 [INSPIRE].

[61] L. Maiani, G. Parisi and R. Petronzio, Bounds on the Number and Masses of Quarks and Leptons, Nucl. Phys. B 136 (1978) 115 [inSPIRE].

[62] A. Andrianov, D. Espriu, M. Kurkov and F. Lizzi, Universal Landau Pole, Phys. Rev. Lett. 111 (2013) 011601 [arXiv:1302.4321] [INSPIRE].

[63] A. Connes, A Unitary invariant in Riemannian geometry, Int. J. Geom. Meth. Mod. Phys. 5 (2008) 1215 [arXiv:0810.2091] [INSPIRE]. 